\title{
COSTRUIRE E VIVERE NEI MONASTERI. MATERIALI E TECNICHE EDILIZIE NEI CANTIERI DI CAMPANIA E MOLISE FRA IX E XII SECOLO
}

\section{ALESSIA FRISETTI}

\author{
UDC: $27-788(450.674) " 08 / 11 "$ \\ $726.71(450.674) " 08 / 11 "$ \\ Preliminary communication \\ Manuscript received: 31. 10. 2016. \\ Revised manuscript accepted: 12. 01. 2017. \\ DOI: 10.1484/J.HAM.5.113741
}

A. Frisetti

Università degli Studi Suor Orsola Benincasa di Napoli LATEM (Laboratorio di Archeologia Tardoantica e Medievale) Via Sorgente, 4, 81016 Piedimonte Matese (Ce)

Italia

The new informations, deduced from the last archaeological excavations in San Vincenzo al Volturno, complete owr knowledges about the organisation of work in the benedictine settlement. The analysis of masonries and the religious buildings census between Northern Campania and Molise, allow us to propose some theories about the diffusion of these technical choices from the IX century to the XII century in this area. Also, in this work we'l prove how the communities of San Vincenzo and Montecassino, contributed to encourage the diffusion of the culture, particularly in the architectural experimentation.

Keywords: medieval masonries; medieval architecture; monastic settlements

L'analisi delle realtà insediative altomedioevali in Campania settentrionale e Molise, condotta in questi ultimi anni dal Laboratorio di Archeologia Tardoantica e Medioevale dell'Università degli Studi Suor Orsola Benincasa di Napoli, ha consentito l'acquisizione di un'importante mole di dati su numerosi insediamenti monastici a cavallo di VIII-X e XI-XII secolo'. La ricognizione ha permesso innanzitutto uno studio delle tecniche e dei materiali edili impiegati nei differenti cantieri con alcuni spot di approfondimento sulle malte, oggetto di analisi chimico fisiche volte alla conoscenza delle ricette e delle fasi di produzione.

Ciò che si vuole presentare in questa sede è il quadro evolutivo di sviluppo e diffusione di alcune tecniche edilizie e soluzioni architettoniche, dimostrando al contempo che due tra i maggiori monasteri dell'Italia centro meridionale, San Vincenzo al Volturno e San Benedetto di Montecassino, hanno evidentemente svolto un ruolo di promozione e diffusione della cultura ed in particolare dell'architettura in un ampio contesto geografico. Il punto di partenza di queste ricerche è stato l'insediamento monastico di San Vincenzo i cui scavi decennali hanno consentito di indagare i molteplici aspetti che riguardano l'architettura monastica ed il cantiere edile nell'altomedioevo ${ }^{2}$.

Alle conoscenze sugli aspetti tecnici delle fasi edilizie che hanno interessato i vari settori del monastero, presentati in più sedi scientifiche nel corso dell'ultimo decennio, si sono aggiunti i dati provenienti dalle indagini più recenti eseguite nell'area lungo la sponda del Volturno e sul Colle della Torre. Riguardo la prima, oggetto di recente pubblicazione, si era già presentata qualche novità nel corso del convegno IRCLAMA del 2015, con particolare riferimento alle tecniche del recinto monastico il cui cantiere è inquadrabile nel corso del IX secolo ${ }^{3}$ (fig. 1).

Lo stesso fronte cronologico sembra aver interessato anche le strutture individuate sul Colle della Torre ad ovest dell'insediamento. In questo contesto le indagini del 20132014 hanno portato alla luce sul pianoro sommitale un imponente edificio, realizzato con grandi blocchi in travertino, ed attraversato da un muro di spina (o mur de refend) con andamento E/O. L'edificio, il cui perimetro non è ancora del tutto noto, accessibile ad ovest dove si conserva una soglia in calcare con alloggio per il cardine della porta, sarebbe stato interessato da un incendio, databile sul finire del IX secolo grazie all'analisi del materiale ceramico e quindi, con buona probabilità associabile all'attacco saraceno dell' 881 . Pertanto la datazione di queste strutture che definiscono un'organizzazione topografica dell'area mai immaginata prima d'ora, è facilmente inquadrabile nella prima metà del IX secolo, in accordo con la fase di maggiore diffusione della tecnica a grandi blocchi tra Lazio e Campania ${ }^{4}$. Allo stato

\footnotetext{
${ }^{1}$ Il lavoro è stato portato avanti di pari passo con lo studio degli insediamenti fortificati e delle relative tecniche costruttive nella Media Valle del Volturno, oggetto della tesi discussa da chi scrive nell'ambito del XXVIII ciclo di Dottorato di Ricerca in Archeologia dell'Università di Roma La Sapienza. Entrambe i lavori sono stati preceduti da una ricerca sugli insediamenti monastici benedettini che la Regione Molise affidò alla cattedra di Archeologia Medioevale dell'Università Suor Orsola Benincasa nel 2010 e i cui risultati sono stati recentemente pubblicati in D. FERRAIUOLO, A. FRISETTI, F. MARAZZI, Medioevo monastico molisano. Atlante degli insediamenti benedettini (VIII-XII secc.), Cerro al Volturno (IS), 2016. Cfr. D. FERRAIUOLO, A. FRISETTI, F. MARAZZI, Medioevo monastico molisano, Cerro al Volturno (Is), c.s.

${ }^{2}$ A. GOBBI, Materiali e tecniche costruttive in un cantiere altomedioevale, in F. MARAZZI, A. GOBBI (a cura di), Il lavoro nella regola. L'approvvigionamento monastico e il cantiere edile di San Vincenzo al Volturno tra IX e XI secolo, Napoli, 2007, p. 83-212; F. MARAZZI, La "Basilica Maior" di San Vincenzo al Volturno (Scavi 2000-2007), Cerro al Volturno (Is), 2014.

3 F. MARAZZI, A. FRISETTI, Porti “monastici" in Campania fra VIII e X secolo, in Hortus Artium Medievalium, 22, Zagreb-Montuvun, 2016, p. 227-237; A. FRISETTI, Le stratigrafie murarie e le tecniche costruttive, in F. MARAZZI, A. LUCIANO, (a cura di), Iuxta Flumen Vulturnum. Gli scavi lungo il fronte fluviale di San Vincenzo al Volturno, Cerro al Volturno (Is), 2015, p. 39-51.

${ }^{4}$ A. FRISETTI, La tecnica a grandi blocchi di reimpiego nella Valle del Volturno, in P. ARTHUR, M.L. IMPERIALE (a cura di), VII Congresso di Archeologia Medioevale, Lecce 9-12 settembre 2105, Firenze, 2015, p. 208-214.
} 


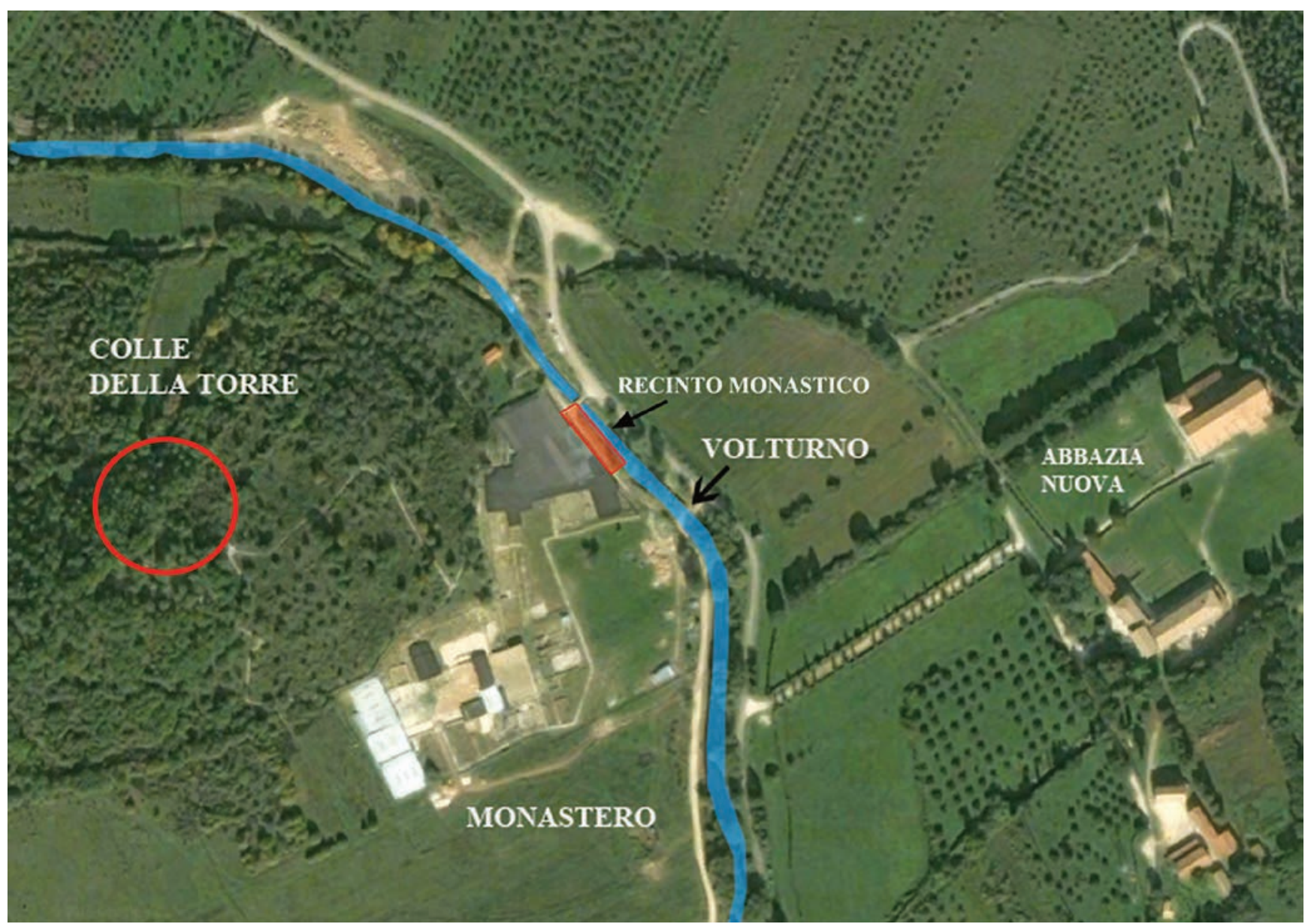

Fig. 1 La piana di Rocchetta al Volturno (IS) e l'insediamento monastico di San Vincenzo. In rosso le aree oggetto di recenti indagini archeologiche

attuale delle ricerche, non è ancora possibile avanzare con certezza la funzione dell'edificio individuato, ma la pianta, le dimensioni dello stesso (14 X $14 \mathrm{~m}$ ca.), il cospicuo spessore delle murature perimetrali ( $1,50 \mathrm{~m}$ circa) e la posizione apicale sull'altura, che consente di traguardare l'intera valle sottostante, lascerebbero pochi dubbi su una sua funzione di carattere difensivo che, tra l'altro spiegherebbe finalmente il toponimo "Colle della Torre" con cui è nota l'area collinare a nord-est dell'insediamento monastico (fig. 2).

Un'ulteriore novità che riguarda sempre il sito vulturnense e che aggiunge un importante tassello per la ricostruzione dell'organizzazione del cantiere edile, proviene da alcuni sondaggi aperti sulla riva opposta del Volturno e propedeutici alla realizzazione di una struttura di accoglienza per i visitatori del sito archeologico.
In particolare, il saggio delta ha permesso di individuare un contesto piuttosto interessante inquadrabile nel corso dell'altomedioevo. Nella zona centrale del saggio è stata rinvenuta una piccola base di forma pseudo circolare leggermente scavata nella superficie ( $\varnothing$ circa $80 \mathrm{~cm}$ e profondità circa $10 \mathrm{~cm}$ ), e collegata sul lato nord ad un condotto verticale a sezione circolare (fig. 3a). Il pessimo stato di conservazione e l'assenza di scorie o prodotti metallici non consentono di avanzare ipotesi certe. Tuttavia, la forma della struttura ed il rinvenimento di frammenti di ganga in tutta l'area, potrebbe far ipotizzare un suo utilizzo come basso forno del tipo a camino ${ }^{5}$. Il condotto verticale di evacuazione, sembra collegato ad un canale scavato nella roccia, profondo in media $50 \mathrm{~cm}$ ed ampio $40 \mathrm{~cm}$, che pure potrebbe essere stato impiegato come canale di deflusso ${ }^{6}$ (fig. 3 b). In questo

\footnotetext{
5 V. LA SALVIA, L'artigianato metallurgico dei Longobardi alla luce delle fonti archeologiche, con particolare riferimento alla lavorazione del ferro. Suggerimenti e problemi, in Archeologia medievale, 25, Firenze, 1998, p. 7-26. Queste strutture diffuse già in epoca pre-classica, presentano una pianta pseudo circolare semiscavata per alloggiare l'alzato in argilla sabbiosa sorretto da una corona in pietre. La cupola così ottenuta, colmata di strati alternati di terra, metallo e carbone, riceve aria da una serie di mantici. Il metallo in forma di bluma si deposita sul fondo del forno, mentre parte delle scorie fuoriesce dal condotto di evacuazione. Per il recupero del metallo si procede poi allo smontaggio della porzione superiore del forno, causa principale tra l'altro del pessimo stato di conservazione di queste strutture e della conseguente difficoltà di riconoscerle in situ (Cfr. S. CABBOI, Ch. DUNIKOWSKI, Les bas fourneaux à scories piégés de la region du Mans, in M. MANGIN (ed.), Le fer, Paris, 2004, p. 56-59; F. ZAGARI Il metallo nel medioevo. Tecniche strutture manufatti, Roma, 2005, p. 65. Tra i casi che possono rappresentare un confronto possiamo citare ad esempio il contesto rinvenuto ad Apigliano e databile al X secolo. Cfr. P. ARTHUR, L. PIEPOLI, L'archeologia del metallo in Terra d'Otranto nel Medioevo, in C. GIARDINO (a cura di), Archeometallurgia: dalla conoscenza alla fruizione, Bari, 2011, p. 243-250). Inoltre, ricordiamo che la semplice forma di queste strutture, rende le stesse adatte a svolgere tanto una funzione di bassifuochi quanto di forge per la raffinazione del blumo (cfr. A. CORRETTI, M. FIRMATI, Metallurgia antica e medievale all'isola d'Elba: vecchi dati e nuove acquisizioni, in C. GIARDINO (a cura di), Archeometallurgia: dalla conoscenza alla fruizione, p. 229-241). Si veda anche G. BIANCHI et alii, La lavorazione del metallo monetabile nel castello di Cugnano (Monterotondo M.mo, GR): lo studio delle aree produttive dei secoli centrali (XI-XII secolo), in F. REDI, A. FORGIONE (a cura di), VI Congresso Nazionale di Archeologia Medievale, L’Aquila 12-15 settembre 2012, Firenze, 2012, p. 644-649.

${ }^{6}$ Sebbene questo canale sia stato rinvenuto completamente riempito di terra e non presentava una copertura in pietre, come è stato invece rilevato in altri casi. A tal proposito si veda l'atelier di Etagnières, cfr. V. SERNEELS, Le travails du fer, in S. REYMOND et alii, La villa romain du Buy et sa forge. Dernières découvertes à Cheseaux, Morrens et Etagnières (Canton de Vaud, Suisse), Lausanne, 2009 (Cahiers d'Achéologie Romande, 115), p. 55-57.
} 

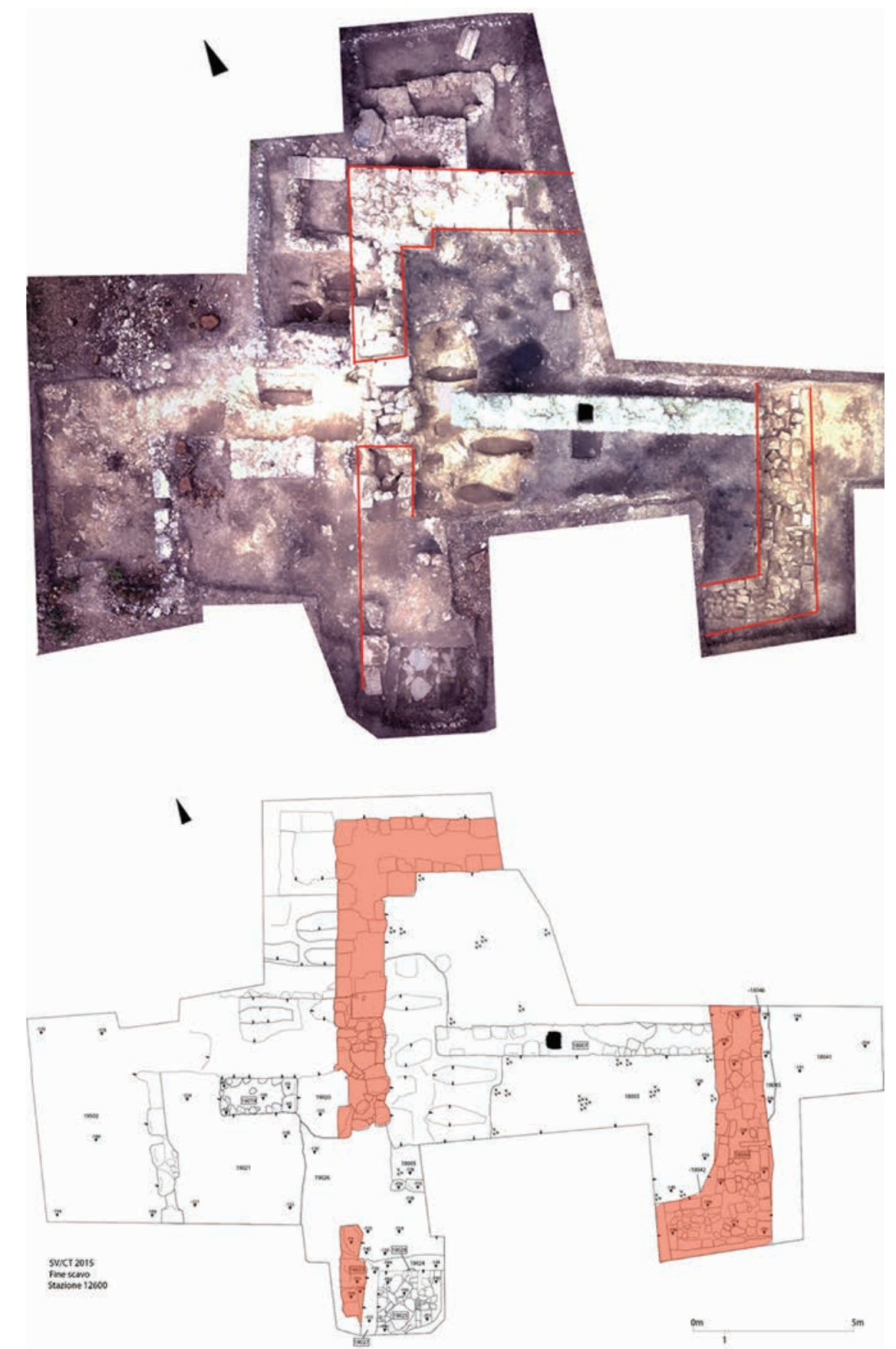

Fig.2 San Vincenzo al V., foto zenitale e rilievo delle strutture sul Colle della Torre (N. Abate, LATEM)

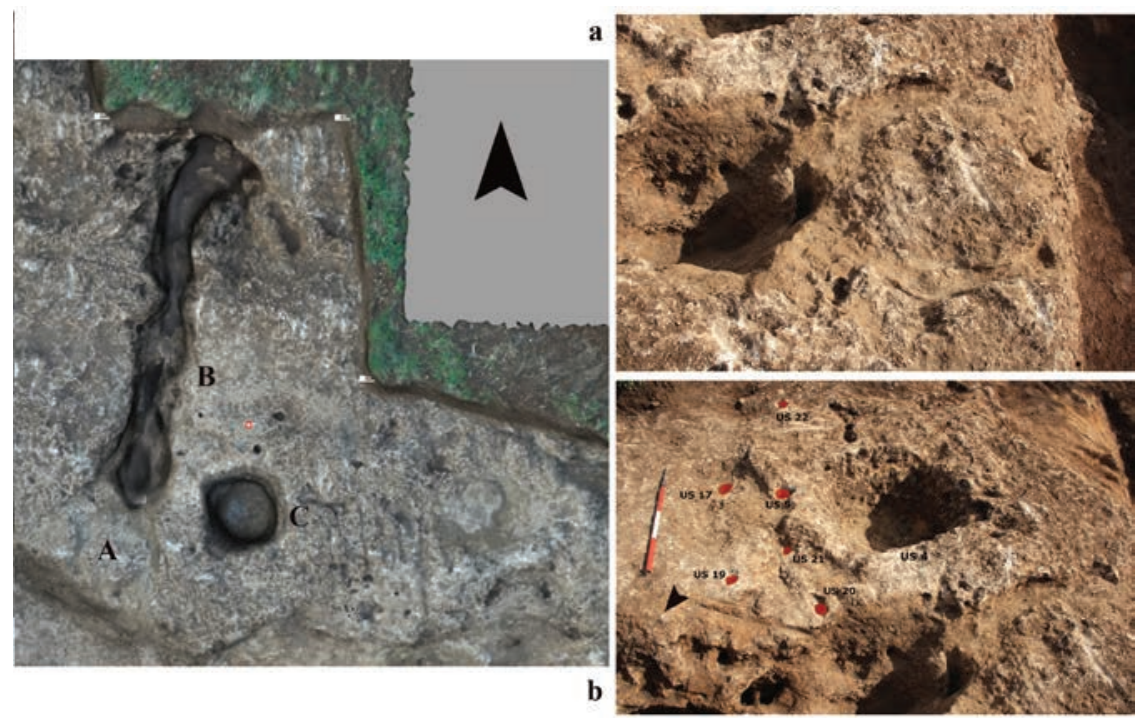

Fig.3 San Vincenzo al V., saggio delta: particolare dell'area produttiva esterna al monastero. a. struttura circolare b. canale di deflusso c. fossa 


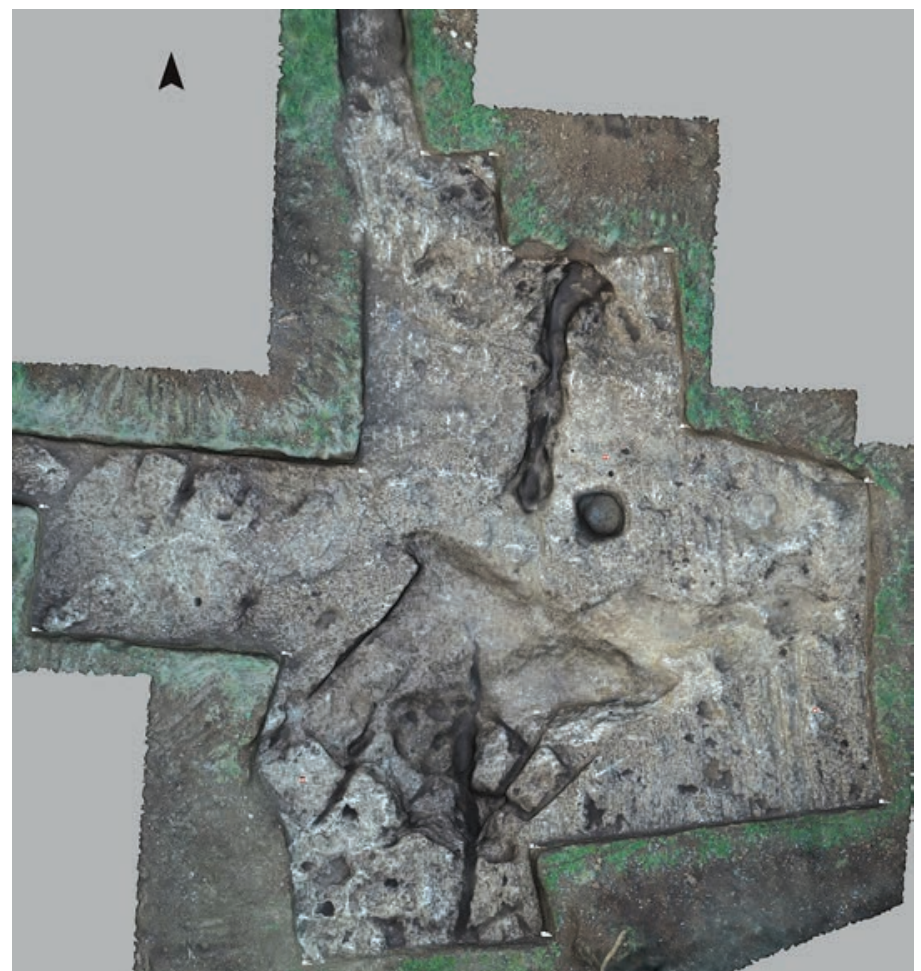

Fig.4 Saggio delta: area produttiva, in basso a destra il piano di calpestio, al centro la zona di cava. (Foto zenitale di N. Abate, LATEM)

caso tuttavia, a differenza di quanto riscontrato in altri siti, l'assenza di indicatori riconducibili all'attività metallurgica, quali ad esempio strati carbonizzati o rubefatti e scorie, impone una certa cautela.

A poca distanza dal forno è stata poi rinvenuta una buca quadrangolare profonda $40 \mathrm{~cm}$ e larga $60 \mathrm{~cm}$, parzialmente colmata da uno strato di carbone e circondata da una doppia sequenza di buche che potrebbero attestare l'esistenza di una piccola copertura a protezione di una probabile fossa di spegnimento (fig. 3c).

Le indagini archeologiche hanno portato alla luce una vasta superficie rocciosa interessata da tracce antropiche anche nella zona sud. In particolare è stato individuato un piano orizzontale (intaccato da interventi agricoli moderni), ottenuto tramite levigatura della superficie rocciosa. Il piano che si presenta particolarmente lisciato e friabile, sarebbe stato realizzato tramite stesura di uno spesso strato di argilla sottoposta a cottura e resa, quindi compatta e funzionale al passaggio di persone e piccoli carri (fig. 4). Una conferma a quest'ipotesi verrebbe proprio dal rinvenimento di numerosi frammenti di argilla con superficie esterna levigata dall'usura e superficie interna piuttosto frastagliata poiché a diretto contatto con la roccia ${ }^{7}$.

Il dato ancor più interessante, è riscontrabile però sul fronte sud-ovest, dove si eseguono tagli longitudinali nella
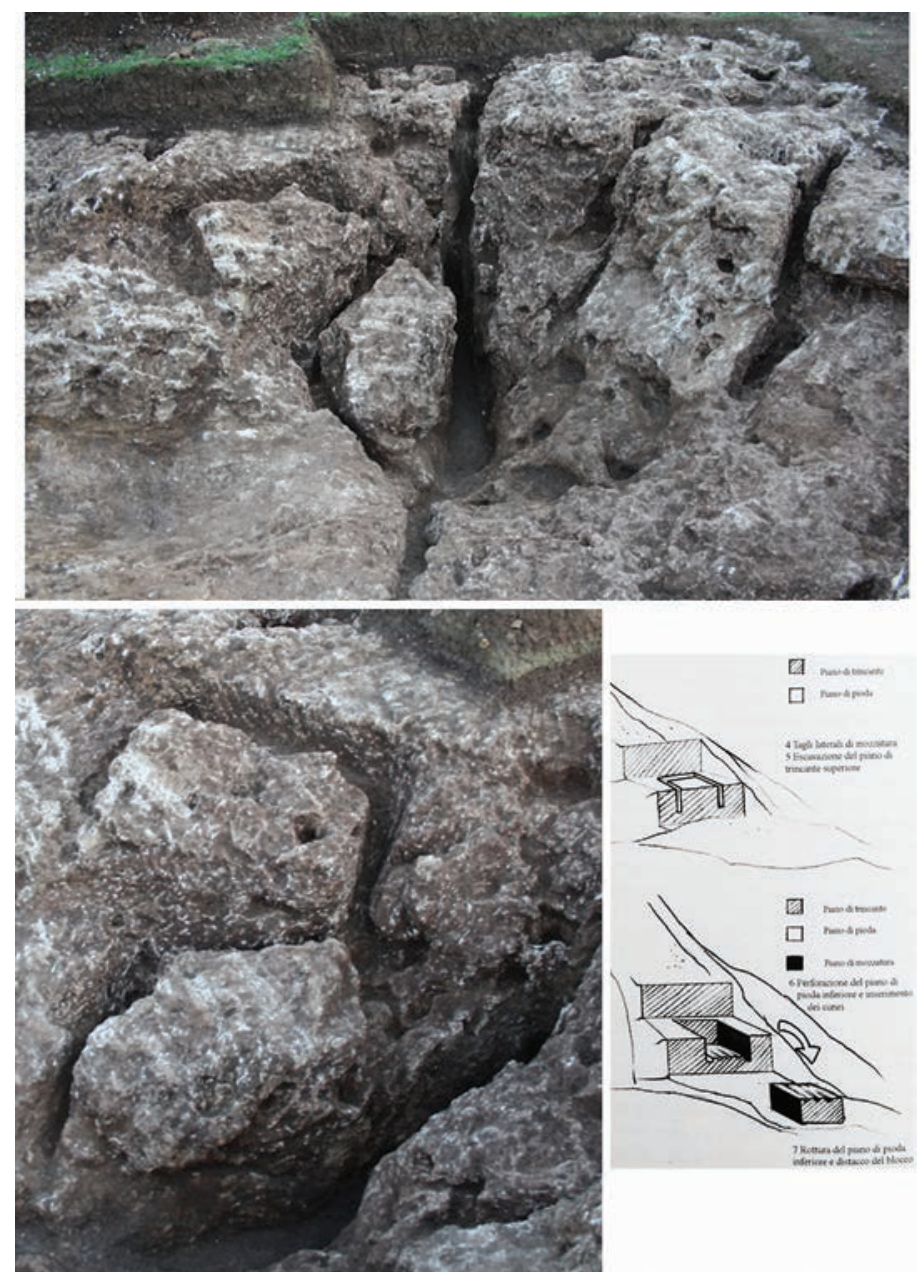

Fig.5 San Vincenzo al V. particolare del fronte di cava e dei blocchi in fase di distacco. A destra ricostruzione del processo di cavatura

(da E. Di Valerio 2010).

roccia (che in parte intaccano anche il forno e il piano di lavoro), ed asportazioni piuttosto evidenti di materiale litico, che suggeriscono la presenza di un fronte di cava (fig. 4). Sono infatti ancora ben visibili alcuni grandi blocchi (lunghi circa $1 \mathrm{~m}$ ) lasciati in situ in fase di distacco, ossia con una superficie ancorata alla roccia emergente. Pertanto, il settore centrale del saggio è interessato da un'ampia area di estrazione che crea una sorta di vasca a pianta trapezoidale, le cui pareti conservano abbastanza bene i segni lasciati dall'uso del piccone (fig. 5). Quest'attività estrattiva deve aver interessato un'area piuttosto ampia, come dimostrano alcuni tagli longitudinali nella zona nord-ovest del saggio, in prossimità della sezione e non del tutto indagati. Si tratterebbe quindi di una cava del tipo "a fossa", tipica delle zone pianeggianti ${ }^{8}$, finalizzata all'estrazione di blocchi, le cui caratteristiche dimensionali sono del tutto simili a quelle riscontrate negli esemplari messi in opera in diversi settori

${ }^{7} \mathrm{~A}$ tal proposito, ma con le dovute cautele del caso, un esempio che si offre come riferimento, anche se pure ancora in corso di studio, è il contesto rinvenuto presso il sito di Miranduolo, interpretato come "quartiere del fabbro", ed inquadrabile in una fase precedente l'edificazione del circuito fortificato di XI secolo. In merito si veda V. FRONZA, Miranduolo (Chiusdino, SI). Il quartiere di un fabbro di VIII secolo, in P. ARTHUR, M.L. IMPERIALE (a cura di), VII Congresso Nazionale di Archeologia Medievale, p. 284-289; V. LA SALVIA, Gli indicatori della produzione metallurgica presso il sito di Miranduolo (Chiusdino, SI) con particolare riferimento alle fasi altomedievali, in F. REDI, A. FORGIONE (a cura di), VI Congresso Nazionale di Archeologia Medievale, p. 640643). Mentre ad un periodo precedente è attribuibile il contesto rinvenuto nei recenti scavi in Piazza Venezia a Roma, dove sono riconoscibili i bassiforni a pozzetto per la riduzione del rame. Cfr. M. SERLORENZI, L. SAGUİ (a cura di), Roma, piazza Venezia. L'indagine archeologica per la realizzazione della metropolitana. Le fasi medievali e moderne, in Archeologia Medievale, 35, Firenze, 2008, p. 175-198. Sul processo di produzione e sui bassiforni si veda L. ESCHENLOHR, V. SERNEELS, Les bas fourneaux mérovingiens de Boécourt, les boulies (Ju/Suisse), Porrentruy, 1991.

${ }^{8}$ E. DI VALERIO, Cenni sui processi di produzione di materiale edile litico nell'antichità, in M.C. SOMMA (a cura di), De Re Monastica II. Cantieri e maestranze nell'Italia medievale, Spoleto, 2010, p. 601-613. 
del monastero. In questi contesti l'estrazione avviene tramite il taglio di 5 facce, mentre il distacco può avvenire per mezzo di cunei in ferro o legno, deformati con l'aiuto di acqua. La presenza di piccoli fori circolari $(\emptyset 3-4 \mathrm{~cm})$ effettuati con trapano e rinvenuti in prossimità degli spigoli dei blocchi in posto, potrebbe invece suggerire la pratica di "markers" per la definizione del posizionamento e delle dimensioni dei blocchi da tagliare ${ }^{9}$.

I dati inerenti l'attività metallurgica e quella di cava consentono di ricostruire quindi, un'organizzazione del cantiere altomedioevale articolata in più fasi. Una prima fase potrebbe prevedere l'estrazione dei minerali, probabilmente la limonite rinvenuta spesso nell'area di scavo del monastero e da cui si ottiene il ferro ${ }^{10}$, le cui tracce sono forse ravvisabili nell'escavazione del grande canale centrale e nell'impostazione dei piani di cottura e della probabile fossa di spegnimento. Tuttavia i pochi dati a disposizione non consentono di chiarire se si tratti di un'area produttiva stabile, o piuttosto di un'istallazione occasionale, che potrebbe essere stata anche finalizzata alla produzione di strumenti (chiodi, cunei, perni ecc.) propedeutici alle operazioni di cavatura.

Segue la fase di cavatura, attestata come abbiamo visto, sia all'esterno del monastero tramite coltivazione in cave a fossa, sia all'interno del perimetro monastico, dove il Colle della Torre è oggetto di interventi che ne trasformano il pendio orientale in un grande fronte di cava a gradoni" ${ }^{11}$. Anche la successiva fase di sbozzatura dei blocchi lapidei avviene tanto in cava quanto nell'area di cantiere, con la possibilità di riutilizzare gli scarti come inzeppature nelle murature. Nella stessa fase potremmo chiaramente inserire anche le operazioni di recupero di spolia con relativa rilavorazione, soprattutto per quanto concerne il calcare ed il marmo ${ }^{12}$.

Questo processo, com'è stato dimostrato in più sedi, soprattutto nel corso del IX secolo, si inserisce in un più ampio sistema produttivo che deve aver visto anche l'edificazione di ponteggi, l'uso di macchine per il trasporto e il sollevamento dei blocchi di grandi dimensioni, nonché la realizzazione di tutte le classi materiali edili con relative fasi di stoccaggio (cui si accennerà a breve). Quanto detto confermerebbe la presenza, probabilmente anche stabile, di maestranze specializzate composte da cavatori, scalpellini, fabbri ed artigiani al completo servizio dell'abbazia tra IX e X secolo.

\section{LA DIFFUSIONE DELLE TECNICHE MURARIE}

Un cantiere monastico così organizzato può facilmente divenire un modello da esportare su scala regionale. In realtà il censimento degli edifici religiosi avviato nell'area a cavallo di Molise e Campania, ha confermato quanto già suggerito dalle fonti scritte ed in particolare dalle cronache monastiche: una relazione diretta fra le comunità di San Vincenzo e Montecassino e la rete costituita da numerosi insediamenti monastici "minori" ad esse direttamente legati, cui si aggiungono altri edifici religiosi in contesti urbani e rurali.

In questa sede si propone pertanto un breve excursus con alcuni esempi pratici, che lungi dall'essere esaustivo, può avvalorare quest'ipotesi. Per quanto riguarda le tecniche murarie, abbiamo visto come l'opera a grandi blocchi sia oggetto di una certa fortuna nel cantiere carolingio di San Vincenzo al Volturno. Questa tecnica viene approntata in diversi settori dell'insediamento, dove si avverte la necessità di impostare fondazioni o sostruzioni atte a risolvere problematiche statiche di diversa natura. Sono queste le esigenze che spingono a costruire le strutture dell'avancorpo antistante l'atrio abbaziale, alcuni pilastri idonei a sorreggere solai di ambienti di collegamento nella zona ovest, i segmenti a dente di sega del recinto lungo il fronte nord del sito a ridosso del fiume ed alcune porzioni della "torre" sull'omonimo Colle. I prodotti appaiono piuttosto diversificati poiché in quasi tutti i casi, il cantiere ha visto l'uso di blocchi di cava associati a materiale di reimpiego di diversa natura (fig. 6).

Questi ed altri esempi del sito vulturnense, sono chiaramente il risultato di soluzioni ingegneristiche di alto livello che qui come a Montecassino, possono essere stati sperimentati, perfezionati e poi adottati anche in altri insediamenti ${ }^{13}$. A tal proposito non può essere quindi un caso se ad esempio nelle strutture superstiti del monastero di San Benedetto in Villa in agro venafrano, si rintraccia la presenza di apparecchi murari in grandi blocchi calcarei di reimpiego, forse provenienti dai vicini mausolei romani. In questo caso l'ispirazione che ha portato a tali soluzioni, e forse la disponibilità di maestranze specializzate, possono essere attribuite tanto ai monaci di S. Benedetto di Montecassino, abbazia madre nelle cui dipendenze ricade l'insediamento di Venafro, quanto alla più vicina comunità vulturnense (fig. 7a).

La diffusione di questa tecnica interessa anche edifici non propriamente monastici, ma attestati tra le dipendenze delle grandi abbazie o rientranti in aree geografiche in cui queste operano. Seguendo questa linea di pensiero, potrebbe essere spiegato quindi il ricorso a paramenti in grandi blocchi di reimpiego anche nell'edificio religioso di San Nicandro e nella cattedrale di Santa Maria entrambi in area venafrana. Nel primo caso, in particolare, la datazione del palinsesto

\footnotetext{
${ }_{9}^{9} \mathrm{~A}$ tal proposito potremmo avanzare anche una seconda ipotesi, sebbene difficilmente dimostrabile. I segni circolari lasciati sulla roccia potrebbero, infatti, essere il risultato di perforazioni superficiali atte ad alloggiare i perni cui legare cavi e funi per facilitare lo spostamento ed il trasporto dei blocchi (cfr. J.P. ADAM, L'arte di costruire presso i Romani, Milano, 1984, p. 30). Un simile sistema, sembra essere stato individuato nel sito di Monte Copiolo (Pu), cfr. D. SACCO, Un manuale per i cavatori inciso nella pietra. Archeologia della produzione lapidea tra X e XII secolo. Le cave di calcare del Castello di Monte Copiolo nel Montefeltro, in Archeologia dell'Architettura,17, Firenze, 2014, p. 191-217.

${ }^{10} \mathrm{Si}$ tratta di una materia prima la cui estrazione è nota già ai Longobardi ben prima del loro arrivo nella penisola italiana. A tal proposito cfr. V. LA SALVIA, L'artigianato metallurgico, op. cit. (n. 5).

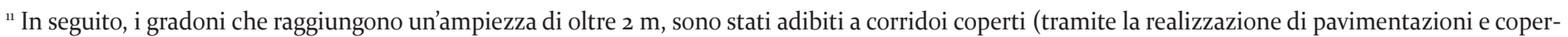
ture), per il collegamento tra ambienti monastici posti a quote differenti.

${ }^{12}$ In merito alle tracce di rilavorazione sui materiali di reimpiego si veda A. CASTELLANI, Riutilizzo e rilavorazione dei marmi romani nell'abbazia altomedioevale di San Vincenzo al Volturno, in II Congresso Nazionale di Archeologia Medioevale (Brescia 200o), Firenze, 2000, p. 304-308; A. FRISETTI, I marmi di reimpiego: materiali architettonici e scultorei, in F. MARAZZI, A. LUCIANO (a cura di), Iuxta Flumen Vulturnum, p. 171-178.

${ }^{13} \mathrm{Nel}$ caso di Montecassino questi paramenti sono stati rintracciati nei resti della torre di Desiderio. Cfr. C. CROVA, Brevi note sui resti della Torre di Desiderio a Montecassino, in M. GIANANDREA, F. GANGEMI, C. COSTANTINI (a cura di), Il potere dell'arte nel Medioevo. Studi in onore di Mario D’Onofrio, Roma, p. 407-418.
} 

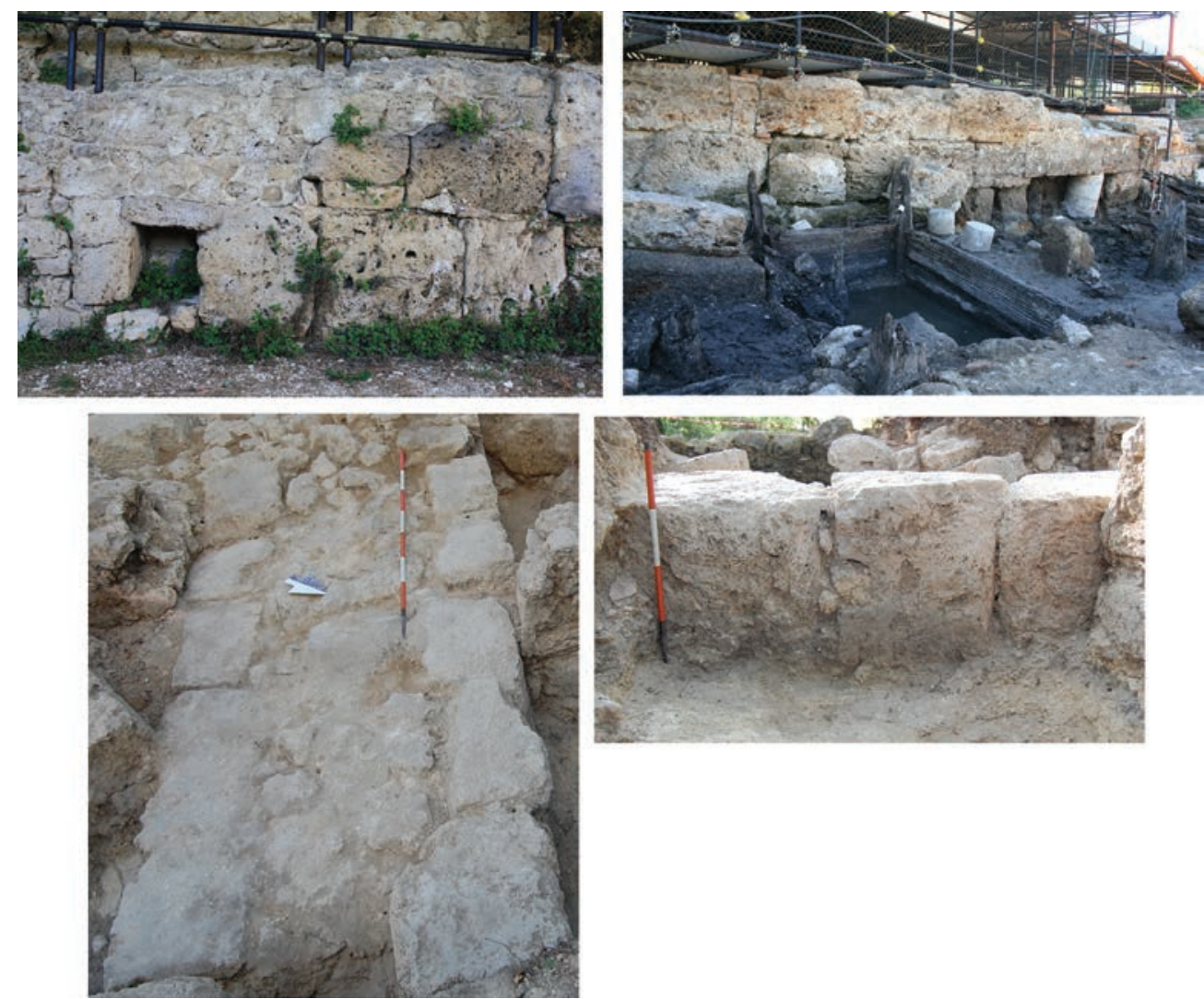

Fig. 6 San Vincenzo al V. Murature a grandi blocchi. Dall'alto in senso orario: avancorpo della basilica, recinto sul fiume, e strutture sul Colle della Torre

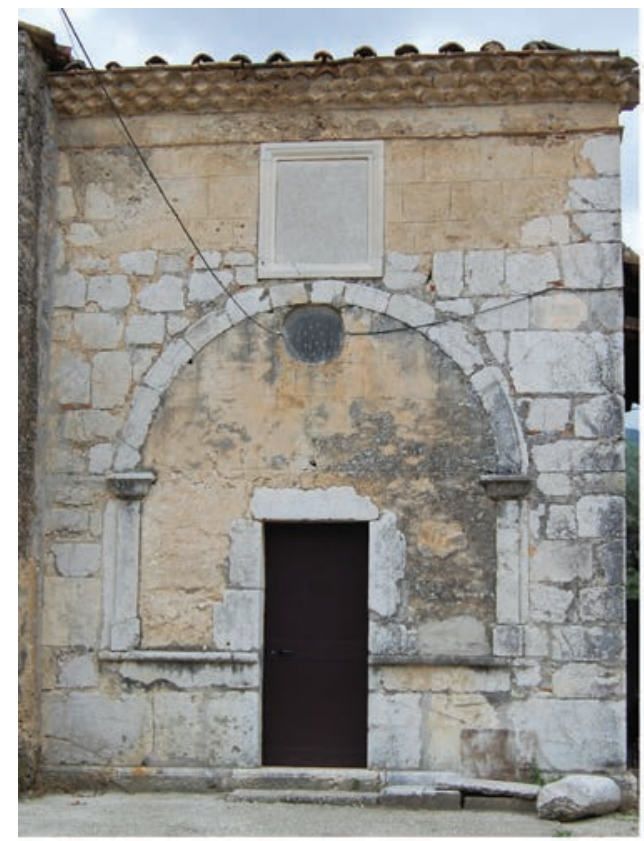

a

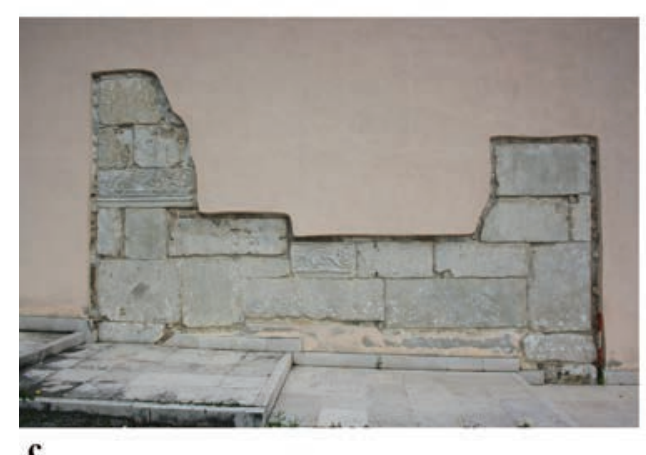

c
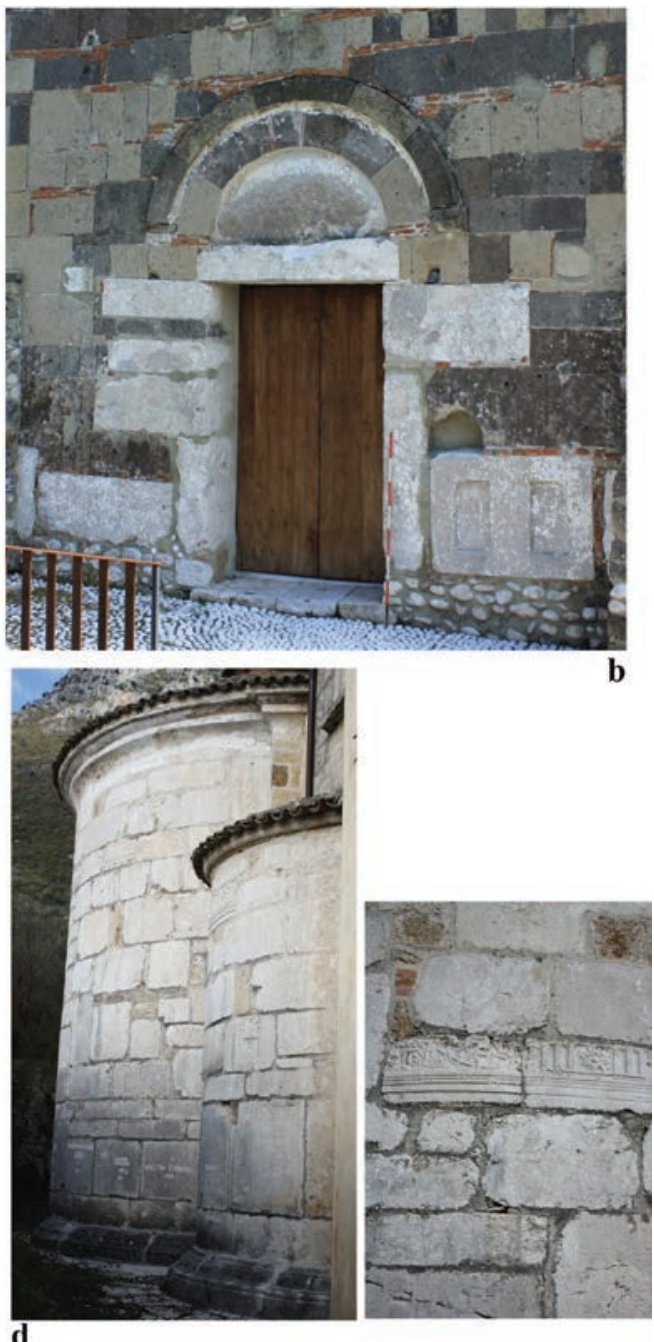

Fig.7 Murature a grandi blocchi. a. San Benedetto in Villa (Venafro, IS); b. Sant'Andrea di Marafi, (Faicchio, BN); c. San Nicandro (Venafro, IS); d. Cattedrale di Santa Maria (Venafro, IS). 
non oltre gli inizi del X secolo ed il rapporto con la comunità monastica vulturnense, sono suggeriti proprio da un documento del 955 contenuto nel Chronicon ${ }^{14}$ (fig. 7c). Anche nel caso della cattedrale extraurbana di Venafro, considerata da molti studiosi un prodotto dell'XI secolo, si propone un simile contesto cronologico, anche perché il paramento in parola è individuabile solo nelle absidi che potrebbero rappresentare proprio una prima fase edilizia della fabbrica ${ }^{15}$ (fig. 7d).

Se poi volessimo spostarci anche in area campana, ed in particolare nella zona settentrionale, potremmo citare il caso della chiesa di Sant'Andrea di Marafi (Bn), dove questa tecnica è impiegata nella porzione della facciata che circonda il portale (fig. 7b). Quest'edificio, rappresenta un esempio particolarmente calzante per le ipotesi appena suggerite in merito al ruolo svolto dai grandi monasteri. La chiesa di Sant'Andrea infatti, si colloca in un contesto territoriale disseminato di beni e proprietà cassinesi evulturnensi, non lontano dall'insediamento fortificato di Castro Porto (loc. Castellone, in comune di Faicchio), citato tra $\mathrm{i}$ possedimenti di San Vincenzo in un documento di XI secolo ${ }^{16}$. Tuttavia bisogna ammettere che, nella zona settentrionale della Campania, certe tecniche siano giunte non solo come risultato dell'influenza esercitata da certe comunità monastiche, ma anche grazie alla febbrile attività edilizia che ha interessato alcuni centri urbani della Langobardia minor, a cavallo di IX e X secolo, quali Benevento, Capua, Cales e Castel Volturno. Ciò giustificherebbe quindi la presenza di paramenti in grandi blocchi di reimpiego, con soluzioni differenti, anche nella fase altomedioevale della basilica di Santa Maria di Compulteria (Alvignano, VIIIIX secolo, fig. 8b), nel basamento della torre campanaria della cattedrale di Telese Terme (per cui pure si propone una datazione al pieno $\mathrm{X}$ secolo, fig. 8c), nei pilastri dell'abbaziale di San Salvatore Telesino (anche questi forse inquadrabili in una fase altomedioevale, fig. $8 \mathrm{a})^{17}$.

L'analisi su vasta scala degli insediamenti religiosi, con particolare riguardo ai siti monastici, ha consen-
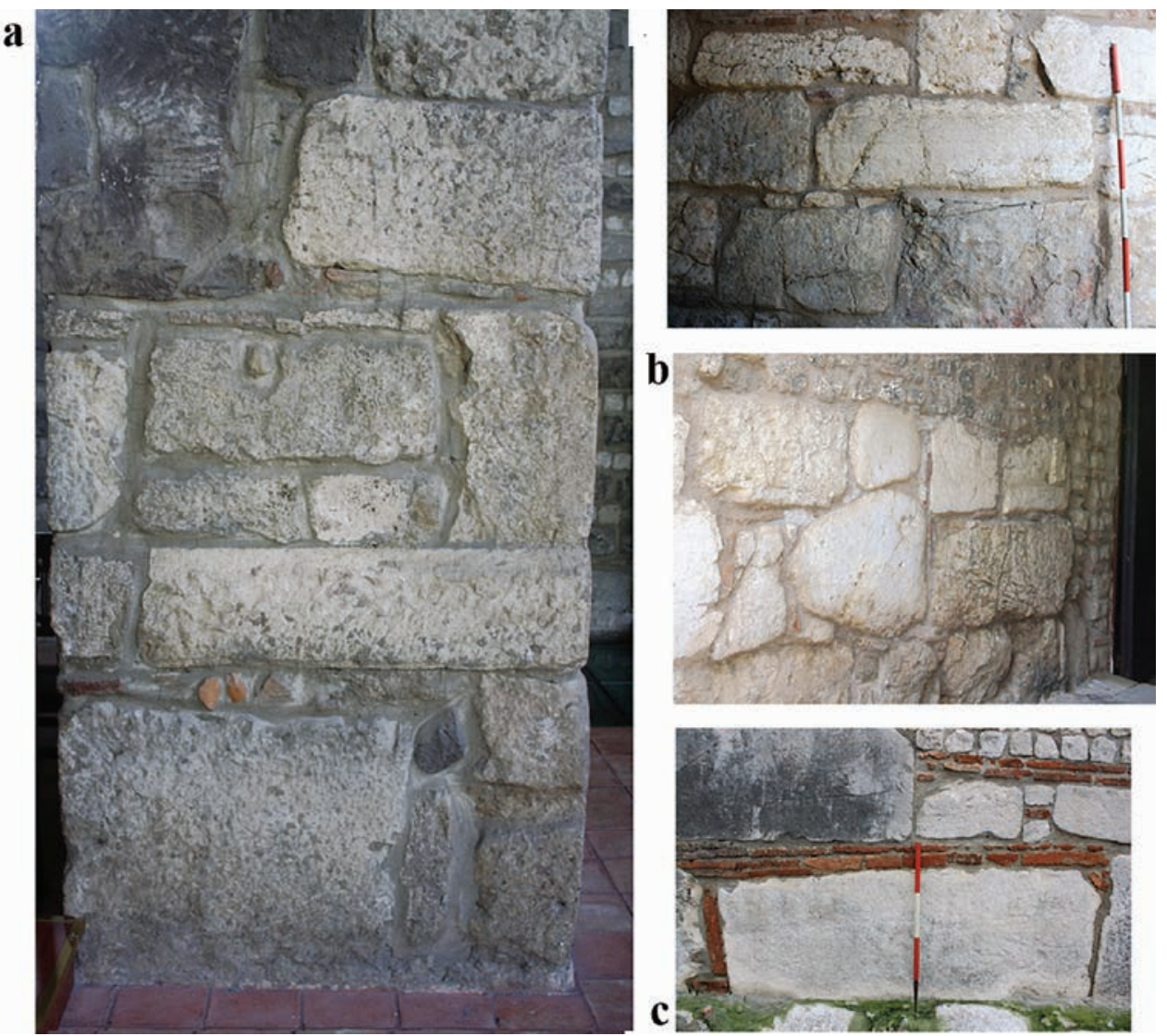

Fig.8 Murature a grandi blocchi. a. Pilastro dell'abbaziale del Salvatore (San Salvatore Telesino, BN); b. cortine interne della torre di Santa Maria di Compulteria (Alvignano, CE); c. basamento della torre campanaria della cattedrale di Telesia (Telese Terme, BN).
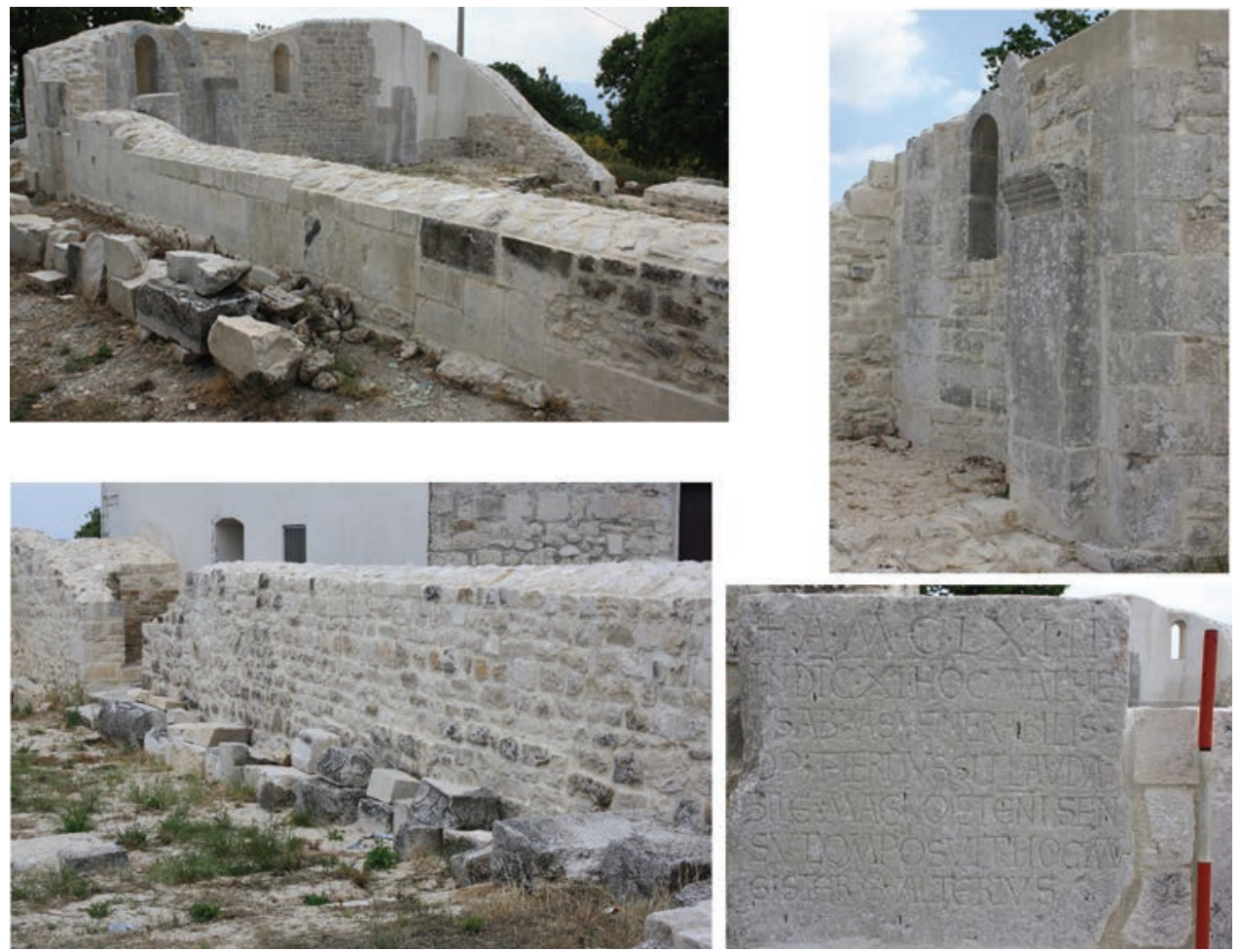

Fig. 9 Santa Maria di Monteverde (Vinchiaturo, CB). In senso orario: perimetrale sinistro; particolare delle absidi; perimetrale sinistro (interno); epigrafe di "Gualterius".

\footnotetext{
${ }^{14}$ Chronicon Vulturnense II, 94.

${ }^{15}$ A. FRISETTI, La tecnica a grandi blocchi di reimpiego, op. cit. (n. 4). Sulle fasi edilizie della cattedrale di Venafro si veda anche G. MARASCO et alii, Le cattedrali di Isernia e Venafro. Il santuario dei Ss. Cosma e Damiano, Isernia, 1993.

${ }^{16} \mathrm{Si}$ tratta di un documento inedito, purtroppo di data incerta, contenuto nel Fondo V. Federici conservato presso la Scuola Speciale Archivisti e Bibliotecari di Roma. Cfr. F. MARAZZI, A. FRISETTI, Porti "monastici", op. cit. (n. 3).
}

${ }^{17}$ A. FRISETTI, La tecnica a grandi blocchi di reimpiego, op. cit. (n. 4). 

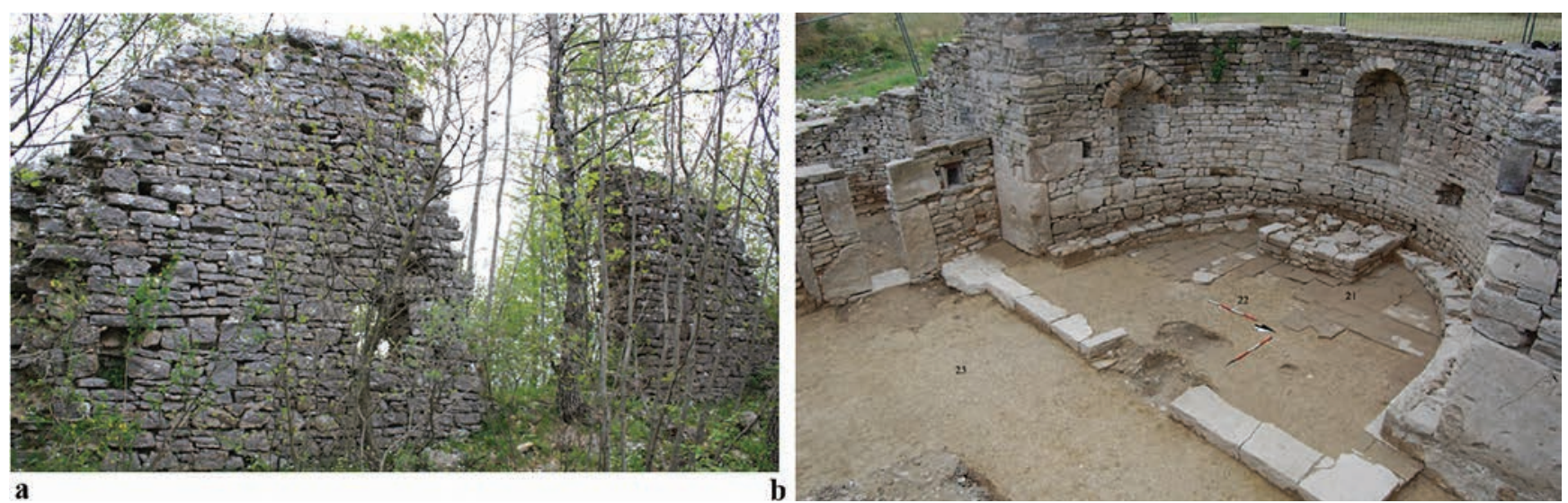

Fig.10 a. Sant'Esutachio ad Arcum (Pietrabbondante, IS), resti della chiesa abbaziale; b. San Benedetto de Iumento Albo (Civitanova del Sannio, IS), veduta della zona absidale (foto D. Ferraiuolo).
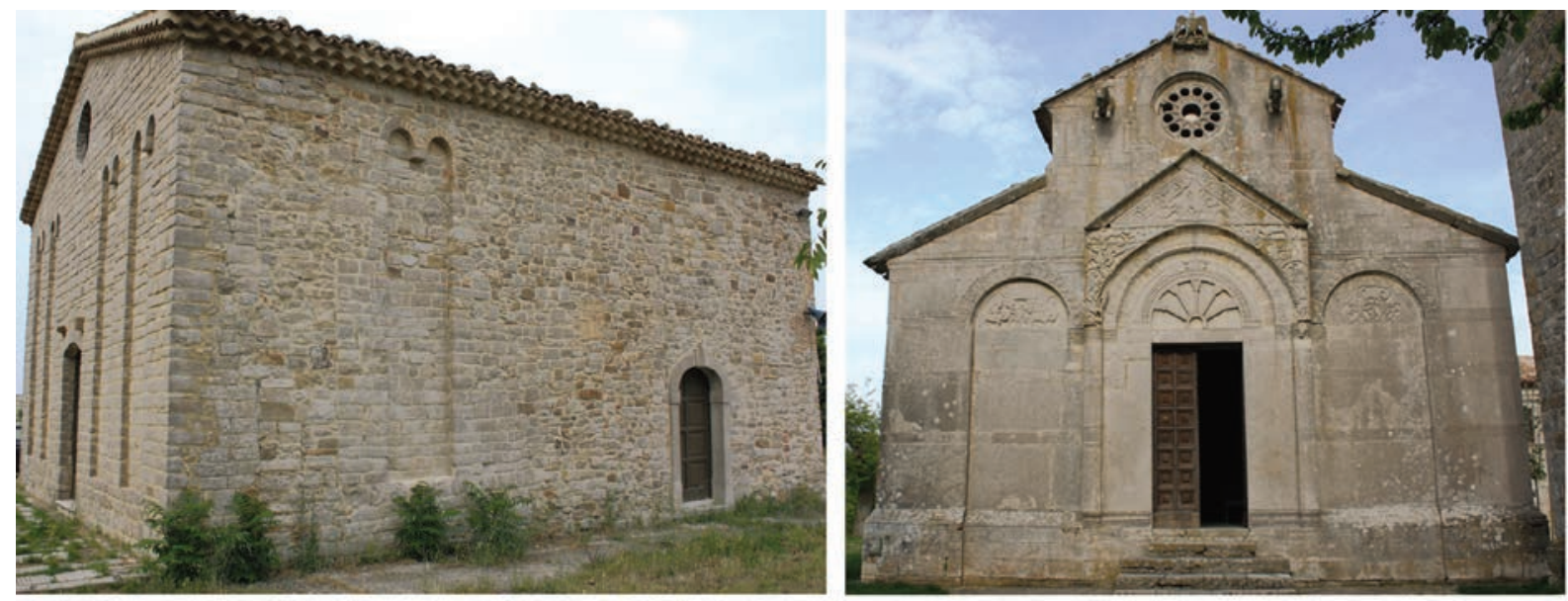

b
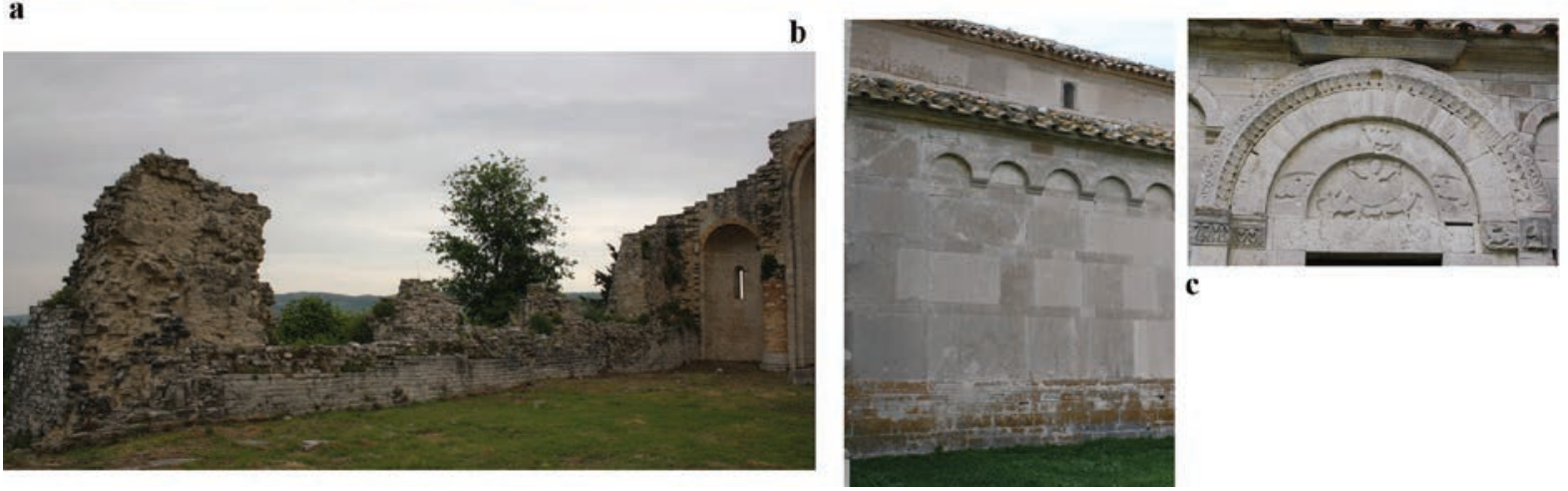

Fig.11 a. Abbaziale di San Pietro (Sant'Elia a Pianisi, CB); b. Santa Maria di Casalpiano (Morrone del Sannio, CB), perimetrale sinistro; c. Santa Maria della Strada (Matrice, CB), facciata e particolari.

tito di ampliare l'arco cronologico ed individuare ulteriori tecniche diffuse tra XI e XII secolo. In particolare, per l'area molisana si nota una certa diffusione di tecniche incerte con pezzame litico di dimensioni medio piccole, spesso associato ad elementi di reimpiego estrapolati da edifici preesistenti sul luogo stesso di fondazione. Dei numerosi casi indagati, ricordiamo ad esempio l'edificio abbaziale di Santa Maria di Monteverde a Vinchiaturo $(\mathrm{Cb})$, dove paramenti a grandi blocchi (nelle porzioni inferiori, nelle angolate e nelle absidi) caratterizzati da una sapiente messa in opera, convivono con cortine in bozze, bozzette e blocchetti, riferibili ad un cantiere monumentale della prima metà dell'XI secolo. L'edificio è poi oggetto di una nuova fase edilizia nel secolo successivo, quando un'epigrafe del 1163 sembra suggerire l'intervento di maestranze specializzate, ed in particolare di un magister Gualterius forse afferente ad una corporazione di lavoratori di pietre (fig. 9).

Con le dovute cautele del caso, data la scarsità di fonti, possiamo citare anche l'insediamento di Sant'Eustachio ad Arcum presso Pietrabbondante (Is), di pertinenza cassinese. I lacerti murari sopravvissuti, verosimilmente pertinenti alla chiesa abbaziale, presentano paramenti in blocchetti parallelepipedi misti a bozze in calcare e posti in filari non sempre regolari. Queste tessiture potrebbero ricondursi ad un arco cronologico a cavallo di XI e XII secolo, a parziale conferma dei documenti rintracciati e risalenti alla prima metà dell'XI secolo e agli anni ' 30 del XII secolo' ${ }^{18}$ (fig. 10a).

${ }^{18}$ CMC II, 16 e Registrum Petri Diaconi, f. 4r, n 97 . Le schede descrittive di questo e degli altri insediamenti benedettini molisani sono in D. FERRAIUOLO, A. FRISETTI, F. MARAZZI, Medioevo monastico, op. cit. (n. 1). 
Un'evoluzione di questa tecnica, che si concretizza nell'impiego di paramenti in blocchetti rettangolari meglio squadrati, si rintraccia in altri edifici monastici. Tra questi ricordiamo ad esempio l'abbaziale di San Benedetto de Iumento Albo a Civitanova del Sannio (Is), sempre di dipendenza cassinese, citata dalle fonti a partire dal 1002 ed oggetto di recenti indagini archeologiche ${ }^{19}$. In quest'edificio si nota l'impiego di blocchetti in pietra locale di dimensioni differenti ma messi in opera in filari piuttosto regolari, sia nell'area absidale che nei perimetrali, dove tra l'altro si riconoscono almeno due fasi edilizie (fig. 1ob).

Un altro caso interessante è la chiesa del monastero di San Pietro a Sant'Elia a Pianisi (XI-XII secolo), di cui è sconosciuta la dipendenza. In quest'edificio si apprezza l'uso della tecnica a blocchetti e bozze in filari non sempre regolari, in associazione al modulo degli archetti ciechi tipici dell'architettura romanica (fig. 11a).

Un maggiore grado di precisione è visibile invece nella prima abbaziale di Santa Maria di Casalpiano (Morrone del Sannio, $\mathrm{Cb}$ ), anche questa di dipendenza cassinese, che potrebbe inquadrarsi a cavallo di XI e XII secolo, ossia prima dell'edificazione dell'abbaziale romanica di XIII secolo, dove la fase di scalpellatura più attenta produce elementi lapidei più omogenei dal punto di vista dimensionale (fig. 11b). Infine, spingendosi fino alla metà del XII secolo, certamente un caso emblematico è rappresentato dalla chiesa di Santa Maria della Strada a Matrice $(\mathrm{Cb})$. Ben noto per i caratteri tipici del romanico e per le sculture del portale e delle lunette, l'edificio si presenta come un perfetto connubio di soluzioni che propongono nel basamento una tessitura a blocchetti rettangolari e nei paramenti corsi di blocchetti impiegati come divisori di grandi blocchi di cava, non disdegnando materiali di reimpiego e giungendo a forme piuttosto evolute delle soluzioni murarie altomedioevali (fig. 11c).

\section{MATERIALI EDILI ED INDICATORI DI PRODUZIONE}

Lo studio delle tecniche, come di consueto è stato integrato con l'analisi dei materiali costruttivi, potendo confermare l'idea generale che un edificio ed un palinsesto architettonico rispecchiano esattamente la natura geologica del luogo in cui si collocano. Ciò si riflette, negli insediamenti dell'area oggetto di interesse, in uno scarso utilizzo dei materiali tufacei, diffusi soprattutto nella zona nord-est della provincia beneventana.

Anche per quanto concerne l'uso dei materiali fittili, si registra una presenza piuttosto ridotta nei palinsesti presi in esame. Un orizzonte più complesso è rintracciabile al momento soltanto a San Vincenzo, dove è attestata una cospicua produzione di laterizi nel IX secolo, votata all'autoconsumo ${ }^{20}$. Questi prodotti (laterizi pavimentali, tegole, coppi, tubuli) dovevano soddisfare il fabbisogno del cantiere monastico nel suo momento di massima attività. Recentemente è stato inoltre possibile constatare che gli esemplari provenienti dall'area del pontile ligneo sul Volturno, confrontati con i laterizi pavimentali ancora in situ, mostrano un'alta percentuale di firme o marche - eseguite con un piccolo punteruolo in legno o tramite impressione digitale - e costituite tanto da elementi decorativi astratti (linee ondulate, zigzag, rombi, cerchi concentrici realizzati con il compasso), quanto da singole lettere o gruppi di esse. Pertanto si è avuto modo di ricostruire per grandi linee il sistema di stoccaggio, grazie all'analisi dei marchi impressi o graffiti sui prodotti, funzionali anche al pagamento a cottimo delle maestranze. Il lavoro di queste ultime era evidentemente controllato dagli stessi monaci della comunità, in un momento in cui, tra fine VIII e IX secolo, si assiste tra l'altro alla rinascita e ad una conseguente diffusione della scrittura anche in ambito amministrativo ${ }^{21}$.

Il materiale costruttivo maggiormente impiegato nei complessi analizzati è il calcare compatto, le cui formazioni sono diffuse su tutto il territorio campano, dove seguono la dorsale appenninica nella zona occidentale, per poi ridiscendere verso i rilievi della penisola sorrentina. In Molise si sviluppa invece, un settore appenninico costituito da stratificazioni di rocce carbonatiche di diverso tipo ${ }^{22}$. Le caratteristiche fisiche intrinseche di questa roccia (resistenza, durevolezza e tenacità), consentono di ottenere prodotti di differenti dimensioni: grandi blocchi, blocchetti, bozze, bozzette, scaglie e materiale di raccolta non lavorato (ciottoli di fiume).

In area molisana è poi il travertino, presente in diffuse placche di diversa estensione, che risulta particolarmente utilizzato nell'area dell'alta valle del Volturno, dove il caso di San Vincenzo, come abbiamo visto rappresenta un ottimo punto di riferimento per lo studio del processo di estrazione e lavorazione di questo materiale. Le indagini effettuate negli ultimi anni in questo sito, infatti, hanno dimostrato che questo litotipo - che caratterizza buona parte della piana di Rocchetta su cui si sviluppa il monastero - viene cavato già da tempi antichi per ottenere tutte le tipologie di pezzature, dai grandi blocchi alle scaglie e scapoli, per finire poi anche macinato nei composti di calce, come hanno dimostrato le analisi chimico-fisiche ${ }^{23}$. Queste ultime (XRDP, SEM,

${ }^{19}$ F. MARAZZI, D. FERRAIUOLO, Indagini archeologiche presso la chiesa abbaziale di San Benedetto de Iumento Albo a Civitanova del Sannio, in P. ARTHUR, M.L. IMPERIALE (a cura di), VII Congresso Nazionale di Archeologia Medievale, p. 142-147.

${ }^{20}$ A. GOBBI, Materiali e tecniche, op.cit. (n. 2).

${ }^{21}$ Per quanto concerne l'analisi dei materiali fittili e del sistema di stoccaggio vulturnense si veda A. FRISETTI, I materiali fittili, in F. MARAZZI, A. LUCIANO (a cura di), Iuxta Flumen Vulturnum, p. 185-194, e relativa bibliografia. Si ricordi tra l'altro che l'ipotesi di un simile sistema di conteggio è stato ipotizzato anche per l'età repubblicana ed imperiale, cfr. A.E. FELLE, Note sui materiali laterizi della nuova Basilica circiforme della via Ardeatina, in Vetere christianorum, 37, 2000, p. 317-336. Infine, in merito all'attività dello scriptorium di San Vincenzo e alla connessione tra la scrittura libraria equella epigrafica (ivi compresa quella su supporti fittili) si veda F. DE RUBEIS, San Vincenzo al Volturno (Isernia), Santa Maria in Stelle (Verona): due casi per due scritture, in F. MARAZZI (a cura di), Archeologia della parola. Percorsi e strumenti per la tradizione della memoria nel Monastero di San Vincenzo al Volturno, Cerro al Volturno (Is), 2012, p. 63-77; F. DE RUBEIS, La scrittura a San Vincenzo al Volturno fra manoscritti ed epigrafi, in F. MARAZZI (a cura di), San Vincenzo al Volturno. Cultura, istituzioni, economia, Montecassino, 1996, p. 21-40; D. FERRAIUOLO Scrivere, impaginare, scolpire. L'officina lapidaria a San Vincenzo al Volturno (secc. VIII-IX), in F. MARAZZI (a cura di), Archeologia della parola, p. 79-10o.

${ }^{22}$ G. AUSIELLO, Architettura medievale. Tecniche costruttive in Campania, Napoli, 20oo; S. AGOSTINI, Le pietre da costruzione e decorative dell'Abruzzo e del Molise, in M.C. SOMMA (a cura di), Cantieri e maestranze nell'Italia medievale, Atti del Convegno di studio, Chieti-San Salvo, 16-18 maggio 2008, Spoleto, 2010, p. 265-278.

${ }^{23}$ A. GOBBI, Materiali e tecniche, op. cit (n. 2). Si veda anche A. VENOSINI, Inquadramento geografico e geologico dell'Alta Valle del Volturno, in F. MARAZZI, A. GOBBI (a cura di), Il lavoro nella regola, p. 77-82; A. MOSCARIELLO, Lo sfruttamento delle risorse naturali, Ibidem, p. 213-224; G. TROJSI, Analisi chimico-petrografiche di alcune malte provenienti dal sito di San Vincenzo al Volturno, Ibidem, p. 225-238. 

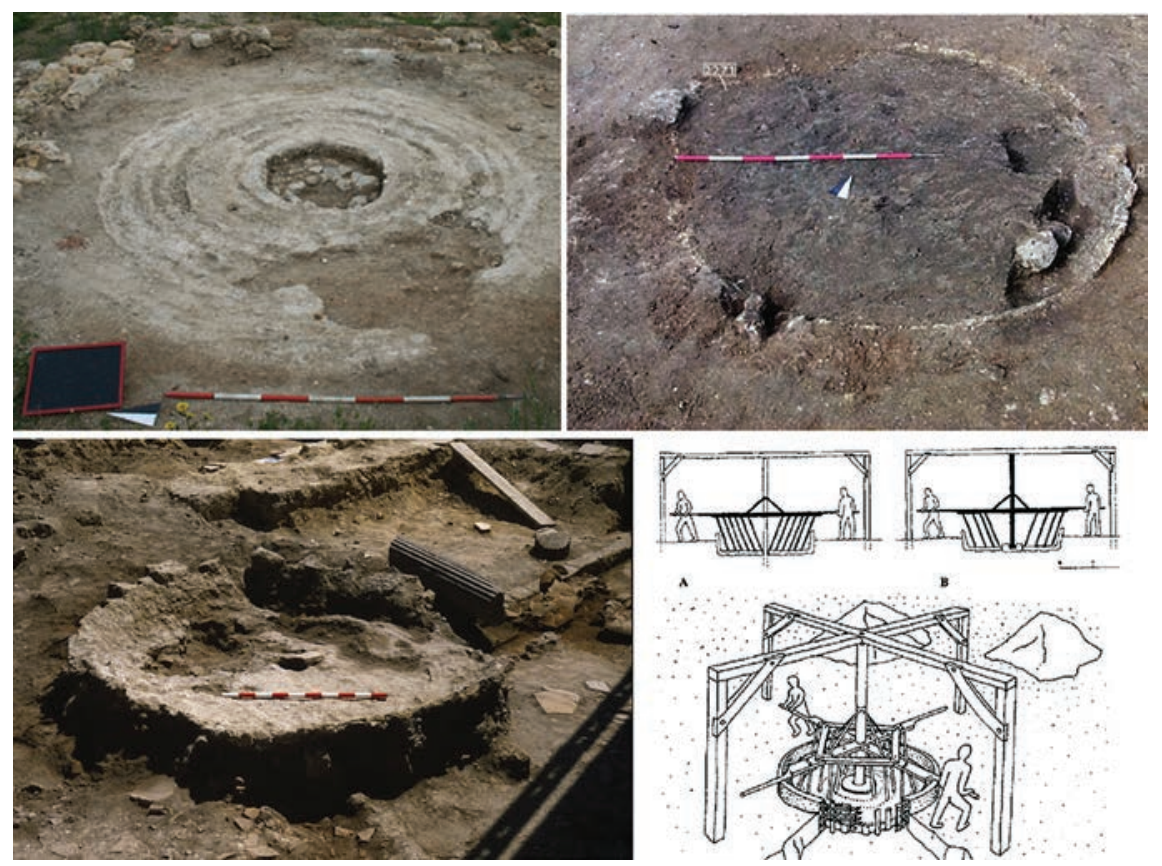

Fig. 12 San Vincenzo al V., esempi di molazze rinvenute in fase di scavo. In senso orario: area $S-E$ del chiostro; area CL/D; giardino porticato; ricostruzione di Gutscher (da G. Bianchi, 2011). di buche ampie e poco profonde e prevedono il ricorso alla trazione animale o umana. Questa, secondo la ricostruzione proposta da Gutscher, consente di azionare un sistema di pali verticali collegati ad un palo centrale più grande di cui spesso si conserva una traccia sul terreno ${ }^{25}$. Nell'insediamento di San Vincenzo sono state portate alla luce ben 6 mole pertinenti ai numerosi interventi edili susseguitisi fra tardo XI e inizi XII secolo. Tra queste ricordiamo la fossa individuata nel Corridoio III quale traccia del cantiere allestito per la risistemazione dell'atrio basilicale ( $\varnothing 3 \mathrm{~m})$; due strutture nel chiostro di cui una (ø $4 \mathrm{~m}$ ) nella zona SO, a testimonianza della fase di smantellamento degli ambienti posti sui corridoi porticati, in direzione della basilica maior; ed un'altra fossa negli strati di abbandono del giardino porticato ${ }^{26}$ (fig. 12). La presenza di queste strutture dimostra l'intervento di maestranze specializzate all'interno del cantiere monastico, a differenza di quanto si registra in altri contesti, dove il ricorso a vasche lignee per la miscelazione della calce non necessita di approfondite conoscenze tecniche ${ }^{27}$.

TGA, sezioni sottili), recentemente ampliate a tutta l'area del Medio Volturno, stanno restituendo dati interessanti. In particolare è stato notato che i campioni contengono in media il $48,9 \%$ di calcite, con picchi che giungono fino al 93\% nei campioni provenienti da edifici romani, anch'essi oggetto di prelievi al fine di confrontare le ricette approntate nel corso dei secoli ed evidenziare eventuali cambiamenti nella composizione e nel processo produttivo. Un esempio peculiare è rappresentato dalla tricora altomedioevale di Campo la Fontana (Capriati a Volturno, Is), posta all'ingresso delle terre vulturnensi in agro venafrano, i cui campioni restituiscono percentuali di calcite tra il $71 \%$ e il 9o\%. In questo caso le percentuali riscontrate confermano l'ipotesi di un cantiere improntato sull'uso di materiali di reimpiego, quasi certamente estratti dal limitrofo ponte romano ancora in uso in epoca altomedioevale ${ }^{24}$.

Le indagini archeologiche degli ultimi anni, hanno inoltre individuato interessanti tracce relative agli indicatori di produzione. Il ciclo produttivo della calce è facilmente ricostruibile ancora una volta a San Vincenzo dove sono stati rinvenuti numerosi miscelatori a pianta circolare (le così dette molazze). Questi sono realizzati tramite lo scavo

\section{CONSIDERAZIONI CONCLUSIVE}

I dati presentati in questa sede descrivono chiaramente un processo di diffusione delle pratiche costruttive che, a partire dai grandi centri monastici quali luoghi di sperimentazione, giungono fino agli insediamenti minori. Se le fonti documentarie sono spesso aride di informazioni, il ricorrere di alcuni caratteri tecnico-architettonici può aiutare a ricostruire una rete di contatti che, attraverso le principali strade romane ancora in uso nel medioevo ed $\mathrm{i}$ nuovi percorsi di pellegrinaggio cristiano, deve aver permesso inevitabilmente scambi di ogni genere tra le abbazie madri e le relative dipendenze dislocate sul territorio. Per tale motivo è facile immaginare che i monasteri come San Vincenzo e Montecassino rappresentino un modello non soltanto per la diffusione delle tecniche ma anche per il replicarsi di sistemi progettuali, impianti planimetrici e corpi di fabbrica che si riscontrano in siti e contesti che in alcuni casi potremmo definire "insospettabili".

Nell'area campano-molisana sono stati individuati alcuni elementi che potrebbero essere stati ispirati dai

\footnotetext{
${ }^{24}$ Le indagini sulle malte che hanno interessato un gruppo piuttosto nutrito di insediamenti post antichi della Media Valle del Volturno, sono state effettuate in tandem dai laboratori del Dipartimento di Chimica dell'Università di Pavia e dal laboratorio di diagnostica e restauro dell'Università Suor Orsola Benincasa di Napoli. I risultati delle indagini sono in parte pubblicati in P. COFRANCESCO et alii, Analysis and characterization of ancient mortars of the Medio Volturno River area, in 1st International Conference on Metrology for Archaeology, Benevento, October 22-23, 2015, p. 484-494.

${ }_{25} \mathrm{Cfr}$. G. BIANCHI, Miscelare la calce tra lavoro manuale e meccanico. Organizzazione del cantiere e possibili tematismi di ricerca, in Archeologia dell'Architettura, 16, Firenze, 2011, p. 9-18.

${ }^{26}$ F. MARAZZI, La basilica maior, op. cit. (n. 2).

${ }^{27}$ È il caso ad esempio delle tracce pertinenti a due vasche rettangolari semiscavate nel terreno, rinvenute nella fase cantieristica tardoantica della chiesa di San Pietro a Massa di Faicchio (Bn). Si tratta di vasche che in questo caso, visti anche i dati preliminari delle indagini sui campioni di malta, potrebbero essere state impiegate soprattutto per la miscelazione del grassello con la sabbia. Tuttavia, la tipologia e la forma rendono questi contenitori adatti anche allo spegnimento della calce viva con acqua (G. FICHERA, Archeologia sperimentale alla Rocca di Sa Silvestro (LI). Dal ciclo di produzione della calce alla costruzione di una casa, in Archeologia medievale, 16, Firenze, 2011, p. 86-95). Gli esemplari di Faicchio, comunque, hanno un buon confronto in alcune tracce rinvenute negli scavi della zona prossima al complesso di San Paolo f.l.m. a Roma, e relative alle fasi altomedioevali di risistemazione dell'area (L. SPERA, L. ESPOSITO, E. GIORGI, Costruire a Roma nel Medioevo: evidenze di cantiere a San Paolo fuori le mura, in Archeologia dell'Architettura, 16, Firenze, p. 19-33). ${ }^{28}$ R.HODGES, S. GIBSON, A.HANSZ, Campo la Fontana: a late eight century triconch and the Ponte Latrone at the entrance to the territory of San Vincenzo al Volturno, in "BAR 58", 1992, p.273-297. I primi esempi monumentali sono applicati in contesti di età adrianea, per poi comparire nelle villae con funzione di triclinia, fino ad irrompere nelle ville rurali nel corso del IV secolo (C. SFAMENI, Ville residenziali nell'Italia tardoantica, Bari, 2006; I. BALDINI LIPPOLIS, Architettura residenziale nelle città tardoantiche, Roma, 2005). Quando lo schema approda in ambito cristiano, è finalizzato spesso alla creazione di strutture martiriali (P. TESTINI, Archeologia cristiana, Bari, 1980).
} 
cantieri di San Vincenzo e Montecassino. Per quanto riguarda i secoli altomedioevali, la presenza ad esempio di due edifici rurali a pianta tricora - uno databile tra VIII e IX secolo, posto a ridosso di un importante ponte romano che attraversava il Volturno (Campo la Fontana, Capriati a Volturno, CE), e l'altro lungo un diverticolo della via Latina presso la città romana di Alife - testimonia la diffusione di un modello di antica tradizione ma già pienamente sperimentato a San Vincenzo nella chiesa nord ${ }^{28}$. Tale ipotesi tuttavia appare più probabile nel caso di Campo la Fontana, vista la prossimità con le terre vulturnensi in agro venafrano. Nel caso di Alife invece, si può forse pensare ad una committenza aristocratica, tutt'ora sconosciuta ma di alto profilo culturale.

Nel primo caso abbiamo un edificio a pianta composita, ossia nata dall'associazione di un vano rettangolare ad ingresso frontale con un ambiente quadrangolare dotato di tre piccole conche absidali. La chiesa di Alife, oggi nota come santuario di Madonna delle Grazie, invece è un esempio di riutilizzo di un antico mausoleo romano di I sec. d.C. con pianta circolare, cui si aggiungono esternamente, ma senza alcun collegamento con il vano interno, tre nicchie semicircolari poco profonde. Queste, che sembrano solo voler accennare all'impianto di un triconco, definiscono un profilo morbido e privo di spigoli che si avvicina molto al caso di Campo la Fontana $^{29}$ (fig. 13). Nel territorio preso in esame, quest'edificio si presenta come un unicum, soprattutto se visto in relazione ad altri mausolei romani, sempre lungo la Via Latina presso Alife, che pur trasformati in chiese non subiscono modifiche strutturali di questo tipo.

Certamente il confronto con San Vincenzo appare suggestivo ma bisogna ammettere che i dati a nostra disposizione non consentono di rispondere alla domanda che ci si pone osservando queste strutture: perché la scelta di una pianta trilobata proprio in questi contesti ed in questo periodo? $3^{\circ} \mathrm{Il}$ rapporto con le grandi abbazie è ancor più evidente se guardiamo alla diffusione o alla trasposizione di altri modelli, quali ad esempio la torre in facciata e l'impianto basilicale a tre navate triabsidate.

Nel primo caso l'adozione in ambito monastico di un modello che fa riferimento al westwerk, suggerisce una dichiarata adesione politica, sebbene l'interpretazione
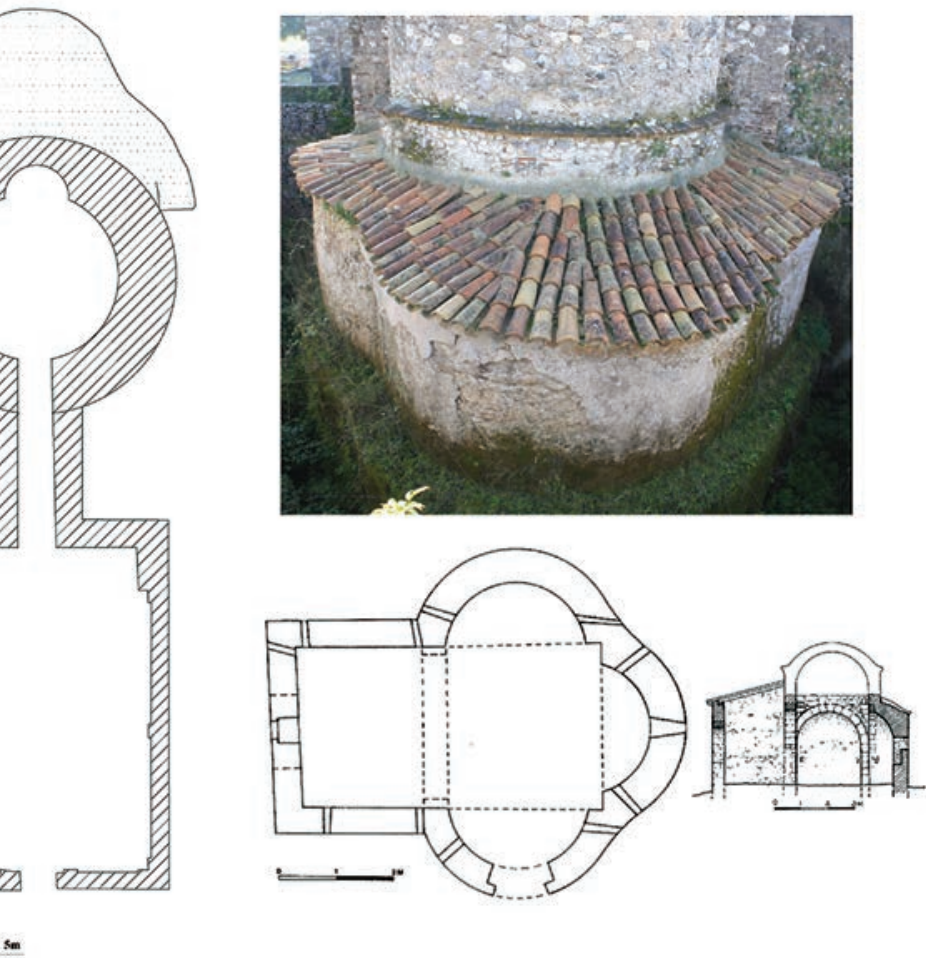

(1)

o pianta e sezione della tricora di Campo la Fontana (da S. Carella 2011).

dell'impianto risulti ormai semplificata nelle forme e nelle dimensioni e svuotata dei suoi significati politici. Questo tipo di strutture che vediamo quasi contemporaneamente a San Vincenzo e a Montecassino ${ }^{31}$, non a caso afferenti a comunità filoimperiali, come ha suggerito Federico Marazzi, avrebbe potuto forse conservare anche una funzione liturgica se visto in connessione con tutto il sistema del quadriportico. Tuttavia, ciò non è possibile nei casi in cui si attesta la presenza della sola torre in facciata. ciò non è possibile nei casi in cui si presenta la sola torre in facciata ${ }^{32}$. Si tratterebbe quindi del risultato di un lungo "metabolismo" che si conclude con un'evidente snellimento del complesso, protraendosi fino agli inizi del XII secolo, ed interessando il Molise, il Lazio Meridionale, la Puglia e la Campania. D'altronde l'ipotesi della semplificazione è stata accertata anche in altri casi, al di fuori della penisola, primo fra tutti la Croazia dove, l'attestazione dei corpi occidentali nel corso del IX secolo, già semplificati nelle loro forme, ha permesso di chiarire come questi siano stati il risultato di una volontaria acquisizione e traduzione in chiave più essenziale dei caratteri architettonici carolingi ${ }^{33}$.

\footnotetext{
${ }^{29}$ In questa sede non è possibile citare tutti gli studi di settore ed i numerosissimi edifici religiosi italiani ed europei che possono rappresentare un confronto con i nostri esemplari, tuttavia si vuole ricordare che ottimi spunti di riflessione sono rintracciabili in S. CARELLA, Architecture religeuse haut-mèdièval en Italie mèridionale: le diocése de Bénévent, Rome, 2011, con relativa bibliografia; A. LUCIANO, Santuari paleocristiani in Italia, tesi di Dottorato di Ricerca in Scienze dell'Antichità, Università degli Studi di Udine, XXV ciclo, 2014; AA.VV. CARE, in Hortus Artium Medievalium, 18, Zagreb-Motovun, 2012; e nella piattaforma digitale WIKICARE in cui stanno confluendo i dati delle ricerche europee del Corpus Architecturae Religiosae Europeae (IV-X saec.).

${ }^{30}$ Sull'edificio di Campo la Fontana si veda S. CARELLA, Architecture religieuse haut-mèdièval, op. cit. (n. 30), p. 71-72. Per le fasi romane della struttura di Alife, invece si veda S. DE CARO, La terra nera degli antichi campani. Guida archeologica della provincia di Caserta, Napoli, 2012. Sugli altri mausolei dell'agro alifano cfr. F. MIELE, Allifae e il suo ager, in F. SIRANO (a cura di), In itinere. Ricerche di archeologia in Campania, Atti del I e del II ciclo di conferenze di ricerca archeologica nell'Alto Casertano, Aversa (Ce), 2006, p. 183-220.

${ }^{31} \mathrm{Ma}$ anche Farfa e Subiaco. Quest'ultima in particolare avrebbe visto la realizzazione della torre sul finire del X secolo, quindi in netto anticipo rispetto agli altri casi (F. BETTI, Da Subiaco a Montecassino. Origine e diffusione della torre in facciata in alcuni edifici religiosi protoromanici del Lazio meridionale, in A. CADEI (a cura di), Arte d'Occidente: temi e metodi. Studi in onore di Angela Romagnoli, Roma, 1991, p. 71-81). Per Montecassino si veda C. CROVA, Brevi note sui resti della torre, op. cit. (n. 13).

${ }^{32}$ F. MARAZZI, La basilica maior, op. cit. (n. 2), p. 333.

33 In merito si veda M. JURKOVIĆ, L'eglise et l'etat en Croatie au IX siecle. Le probleme du massif occidental carolingien, in Hortus Artium Medievalium, 3 , Zagreb-Motovun, 1997, p. 23-40, in cui si affronta la diffusione e l'evoluzione di queste strutture a cavallo di IX e XI secolo.
} 


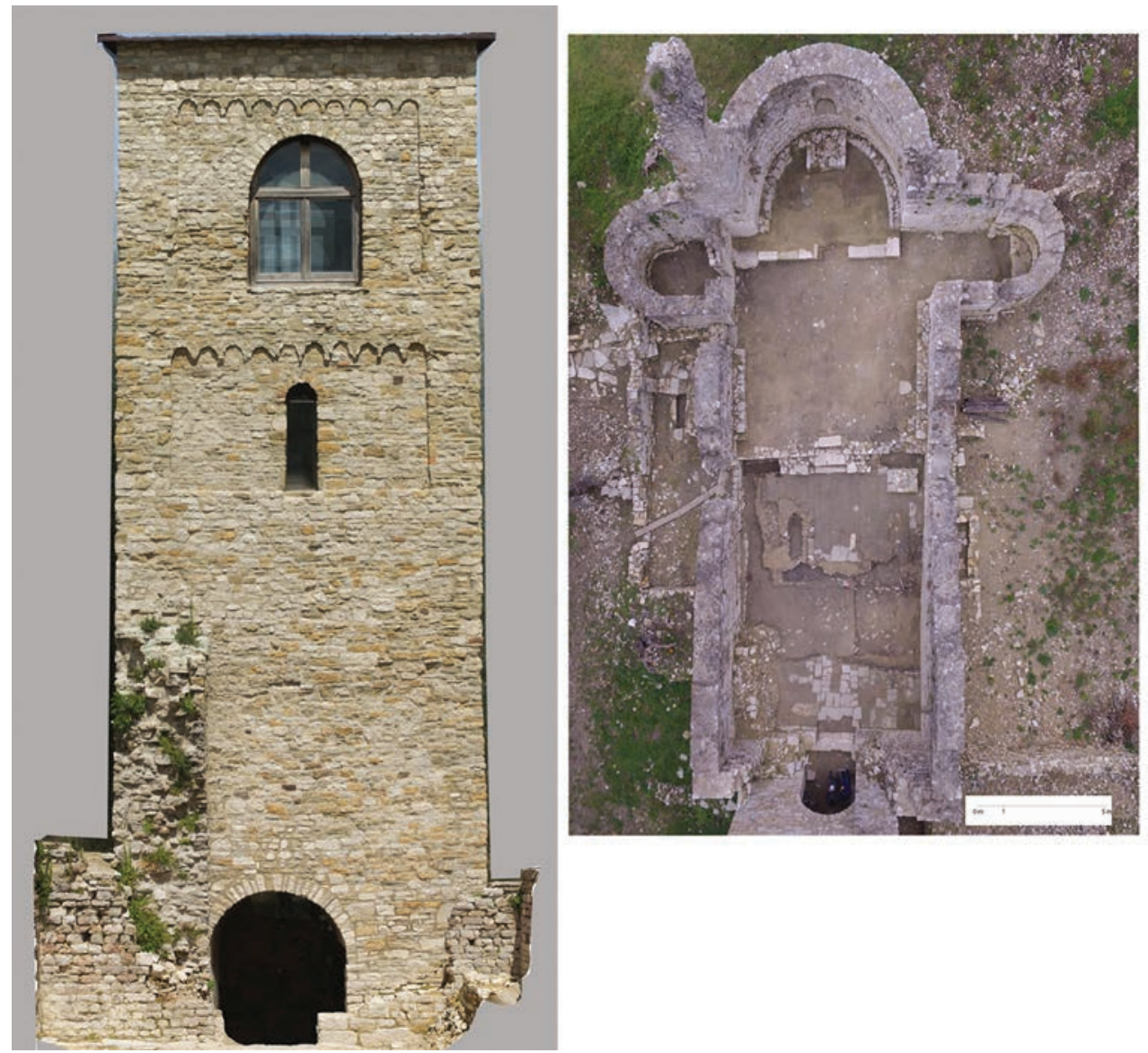

Fig. 14 San Benedetto de Iumento Albo (Civitanova del Sannio, IS), fotopiano della torre in facciata vista dall'interno e foto zenitale dell'edificio abbaziale (N. Abate, LATEM). di Civitanova ${ }^{34}$. Le recenti indagini archeologiche, hanno infatti dimostrato che la torre rappresenta una fase edilizia successiva. La struttura turrita, infatti, "si aggancia" all'edificio religioso tramite l'innesto di due pilastri-contrafforti che di fatto colmano lo spazio venutosi a creare tra i due corpi di fabbrica (fig. 14). I confronti tipologici rimandano, come già accennato, ad alcuni dei principali edifici monastici della penisola, cui possiamo aggiungere anche le chiese abbaziali di San Pietro alla Foresta, Santa Maria di Correano, Santa Maria di Castagneto presso Maranola, tutte nel Lazio centro-meridionale e risultato probabilmente dell'influenza delle grandi abbazie benedettine ${ }^{35}$. Ma potremmo spingerci anche oltre i confini italiani per rintracciare confronti in aree limitrofe come la Croazia dove, edifici come Sant'Elia di Bale e Sant'Elia a Valle presso Rovigno presentano strutture turrite del tutto simili con il ricorso ad archi ciechi tipici del romanico che riscontriamo, seppur con caratteri diversi anche a Civitanova del Sannio ${ }^{36}$.

L'accettazione e la successiva cristallizzazione di queste strutture è ancora più evidente se consideriamo anche altri edifici non monastici quali la basilica di Santa Maria di Compulteria ${ }^{37}$, le chiese di San Tomeo (Capriati a Volturno) ${ }^{38}$, Sant'Anastasia a Ponte (Bn), Ss. Rufo e Carponio a Capua, e con qualche probabilità anche gli edifici parrocchiali di Ciorlano (San Nicola) e Piedimonte Matese (San Giovanni) che rientrano nel vasto gruppo degli esemplari caratterizzati da campanili in facciata con porta d'ingresso ${ }^{39}$ (fig. 15).

Per quanto riguarda invece la diffusione dell'impianto basilicale, anche se non è questa la sede per approfondire tale tematica, va comunque ricordato che questo schema approntato a San Vincenzo e Montecassino è oggetto di una certa fortuna a cavallo di VIII e IX secolo come dimostrano i tanti edifici diffusi nella penisola. Per San Vincenzo in particolare, come è stato già suggerito recentemente, la pianta

\footnotetext{
${ }^{34}$ F. MARAZZI, D. FERRAIUOLO, Indagini archeologiche, op. cit. (n. 19); Gattola, Historia 207; CMC II, 32.

35 F. BETTI, Da Subiaco a Montecassino, op. cit. (n. 31).

${ }^{36}$ In merito all'edificio di Bale cfr. M. JURKOVIĆ, L'architecture du premier age roman en Croatie, in Hortus Artium Medievalium, 6, Zagreb-Motovun, 2000, p. 83-92. La chiesa di Sant'Elia in Istria, è invece discussa in S. MUSTAC, M. BARADA, La chiesa di S. Cecilia presso Rovigno-considerazioni prima del restauro, in Hortus Artium Medievalium, 10, Zagreb-Motovun 2004, p. 237-244. L'impianto planimetrico di San Benedetto tra l'altro trova un buon confronto nella chiesa di San Salvatore a Cettina in Dalmazia (A. MILOŠEVIĆ, Z. PEKOVIĆ, Predromanicka crkva Svetoga Spasa u Cetini/ La chiesa preromanica di San Salvatore a Cettina, Dubrovnick-Split, 2009).

${ }^{37}$ A. FRISETTI, La Basilica di S. Maria di Compulteria in Alvignano (Ce): nuove ipotesi di datazione della Ecclesia Cubulterna, in A. COSCARELLA, P. DE SANTIS, Martiri, santi, patroni: per un'archeologia della devozione, Atti X Congresso Nazionale di Archeologia Cristiana (Università della Calabria, 15-18 settembre 2010), Arcavacata di Rende (Cs), 2012, p. 723-730. Per la chiesa di Sant'Anastasia di Ponte, cfr. M. ROTILI, Alle origini di un centro rurale nel Principato longobardo di Benevento: dalla chiesa al castello di Ponte. La chiesa di S. Anastasia, in Campania Sacra: studi e documenti, 8-9, 1977, p. 5-37.

${ }^{38}$ Quest'edificio tra l'altro potrebbe anche essere inserito in un insediamento monastico, ma purtroppo le fonti non consentono di avvalorare quest' 'ipotesi, ma piuttosto di ricordare che esso ricade in un ambito territoriale interessato da proprietà vulturnensi e cassinesi (Chronicon Vulturnense I, p. 372-373).

39 S. LOMARTIRE, L'organisation des avant-corps occidentaux. A propos de qualques exemples de l'Italie du Nord au Moyen Age, in C. SAPIN (ed.), Avantnefs \& espaces d'accueil dans l'église entre le IV et le XII e siecle, Paris, p. 351-371. A questi chiaramente vanno aggiunti i numerosi edifici (chiese, cattedrali) conosciuti in Italia nel periodo compreso fra XI e XII secolo per i quali sarebbe impossibile in questa sede sintetizzare la bibliografia di riferimento.
} 

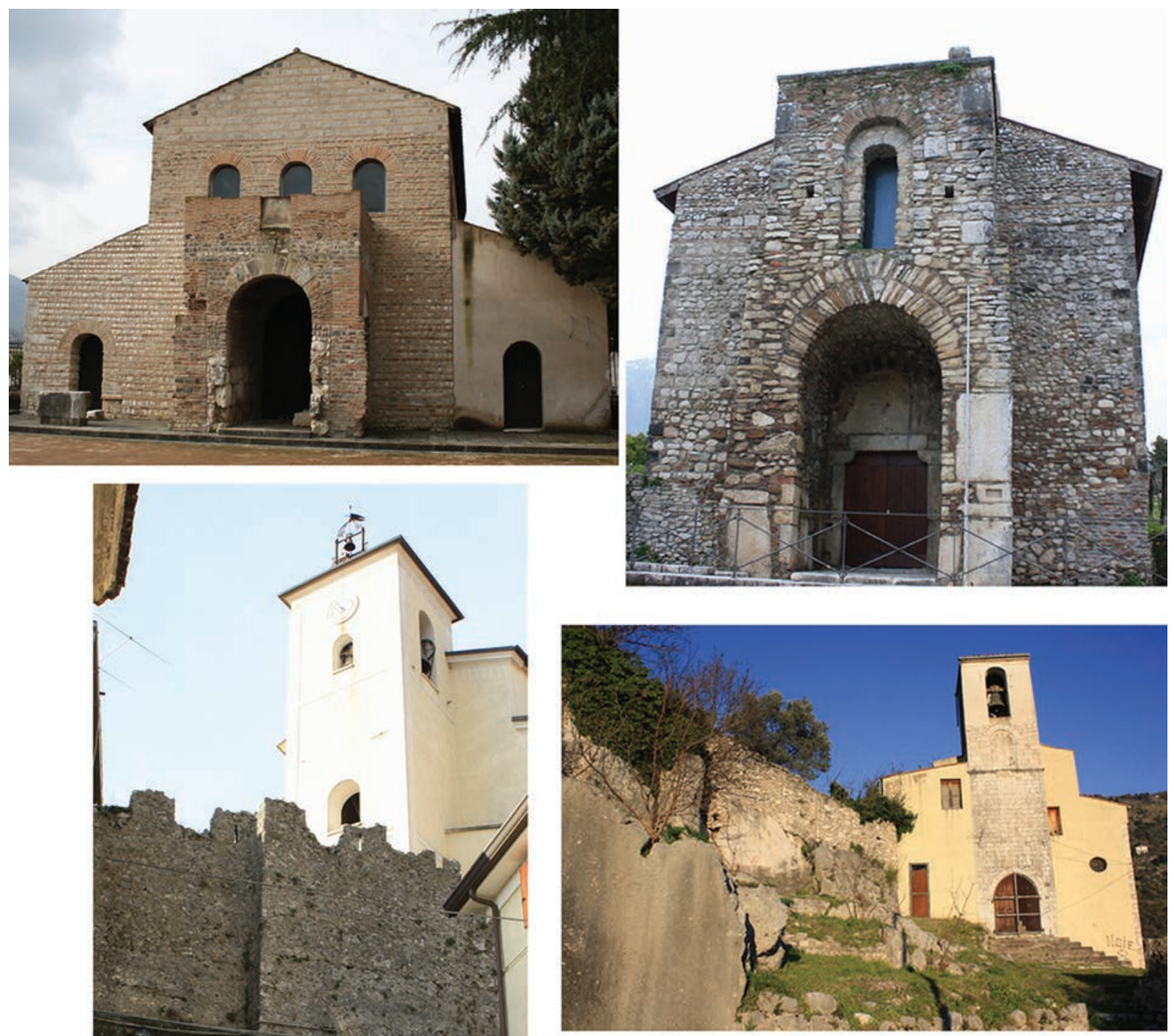

Fig.15 Edifici con torri in facciata. In senso orario: Santa Maria di Compulteria (Alvignano, CE); Sant'Anastasia a Ponte (Ponte, BN); San Giovanni (Piedimonte Matese, CE); San Nicola (Ciorlano, CE).

a tre navate triabsidate potrebbe essere stata determinata dalla necessità di collocare più altari, anche in relazione alle nicchie della cripta che dovevano ospitare, oltre alle reliquie del martire di Saragozza anche quelle di altri santi ${ }^{40}$. Si tratta in ogni caso di un cantiere imponente che rifletterebbe, nel quadro della politica culturale europea, l'ambizione alla grandiosità, più di quanto non venga fatto a Montecassino.

Ma l'ambizione è insita soprattutto nel progetto generale dell'edificio e nel suo collegamento con gli altri corpi di fabbrica dell'insediamento monastico. La diffusione su larga scala geografica di questo impianto tuttavia, è attribuita come è noto alla fortuna del così detto "modello desideriano" sul quale si sono concentrate le analisi di autorevoli studiosi ${ }^{41}$. Questo modello è frutto dell'intervento dell'abate Desiderio che sceglie di conservare l'originario impianto voluto da Gisulfo nel cantiere carolingio, epurandolo delle superfetazioni ottoniane, nel tentativo di recuperare un prototipo paleocristiano ${ }^{42}$.

Nel nostro territorio, per concludere vogliamo ricordare alcuni casi che riprendono proprio questo tipo di impianto seppur con alcune varianti al tema originario. Esempi interessanti sono: la chiesa abbaziale del Salvatore a San Salvatore Telesino, fondata negli anni ' 70 dell'XI secolo e che vede l'introduzione del transetto sporgente (in luogo di quello continuo); la chiesa del monastero cassinese di Santa Maria in Cingla per quanto concerne la fase di XII secolo, ma anche edifici non monastici quali le cattedrali di Telese Terme (Bn, fine XI secolo), Venafro (Is, fase di XI-XII secolo) ed Alife (Ce, anni '3o del XII secolo). Queste ultime in particolare si pongono come risultato della combinazione di due elementi culturali: il modello desideriano e i temi dell'architettura romanica, la cui presenza riflette una ripresa di concilianti rapporti fra la comunità di Montecassino e i Normanni. In questo momento infatti, Desiderio è mosso dalla necessità di adeguare le fabbriche monastiche al ruolo politico che sta ricoprendo il cenobio, schierato con i papi della riforma ed alleato con i Normanni ${ }^{43}$. In tal modo, quindi, si spiegherebbe perché proprio l'abbaziale cassinese diviene modello di riferimento per le più importanti fondazioni normanne soprattutto in Campania, quali ad esempio la cattedrale di

\footnotetext{
${ }^{40}$ In merito si veda F. MARAZZI, La basilica maior, op. cit. (n. 2).

${ }^{41}$ Tra questi si ricordi ad esempio G. CARBONARA, Iussu Desiderii. Montecassino e l'architettura campano-abruzzese nell'XI secolo, Roma, 2014.

${ }^{42}$ G. CARBONARA, Iussu Desiderii, p. 64.

43 Ibidem, p. 25.
} 
Sessa Aurunca, il duomo di Ravello, di Sant'Agata de Goti e di Caserta Vecchia (quest'ultimo con transetto sporgente).

Inoltre va ricordato che nello stesso frangente, proprio a seguito della conquista normanna, anche la tecnica a grandi blocchi riceve un nuovo impulso e si diffonde in Italia meridionale ${ }^{44}$. In questo caso, a differenza del periodo altomedioevale, la nuova compagine culturale alloctona diffonde nuove conoscenze sul piano cantieristico, consentendo la ripresa delle attività di coltivazione dei fronti di cava, che si riflette spesso, in cantieri dall'andamento regolare, con blocchi cavati e lavorati in forme e dimensioni omogenee - in aggiunta agli elementi di reimpiego sempre presenti quando è il calcare il litotipo più frequente - e che contraddistinguono gli edifici religiosi appena citati (ma anche alcune strutture fortificate). $\grave{E}$ in questo quadro, quindi che si inserisce Desiderio abate-architetto, che percepisce la portata delle innovazioni tecniche ed apre una nuova stagione culturale in cui il monastero cassinese è nuovamente centro propulsore di rinnovati linguaggi artistici. 